\title{
SELF-MAPS OF LOOP SPACES. I
}

\author{
BY
}

\author{
H. E. A. CAMPBELL ${ }^{1}$, F. P. PETERSON ${ }^{2}$ AND P. S. SELICK ${ }^{3}$
}

\begin{abstract}
In this paper we study self-maps of $\Omega^{k} S^{m+1}$ and show that, except for the cases $m=1,3,7$, or $p$ odd and $m$ odd, if $f$ induces an isomorphism on $H_{m+1-k}\left(\Omega^{k} S^{m+1} ; Z / p Z\right)$ with $k<m$, then $f_{(p)}$ is a homotopy equivalence.
\end{abstract}

Introduction. In [CM], Cohen and Mahowald prove the following

TheOREM (COHEN-MAHOWALD). Let $f: \Omega^{2} S^{2 n+1} \rightarrow \Omega^{2} S^{2 n+1}$ be a self-map which induces an isomorphism on $H_{2 n-1}\left(\Omega^{2} S^{2 n+1} ; Z / p Z\right)$ with $n>1$. Then $f_{(p)}$ is a homotopy equivalence.

Motivated by this example, we studied the problem of whether this type of result is true for $\Omega^{k} S^{m+1}$ for other values of $k$ and $m$ with $k \leqslant m$. The Hopf fibrations immediately give splittings

$$
\Omega S^{2 n} \approx S^{2 n-1} \times \Omega S^{4 n-1}
$$

if either $n=1,2$, or 4 or if we localize at an odd prime. Thus this result cannot be true if either $m=1,3$, or 7 or if $p$ is odd and $m$ is odd. In addition

$$
\Omega^{m} S^{m+1} \approx S^{1} \times \widetilde{\Omega^{m} S^{m+1}}
$$

so it also fails for $k=m$.

In this paper we show that the analogue of Cohen and Mahowald's theorem holds in all other cases. Roughly speaking, the main theorem states that (with the exceptions noted above) a degree one self-map of $\Omega^{k} S^{m+1}$ is a homotopy equivalence. We show this by proving a stronger statement.

THEOREM 5.1. Let $f: \Omega_{0}^{m+1} S^{m+1} \rightarrow \Omega_{0}^{m+1} S^{m+1}$ be a loop map which induces an isomorphism on $\mathrm{H}_{2 p-3}\left(\Omega_{0}^{m+1} S^{m+1} ; Z / p Z\right)$. If $p>2$, we suppose in addition that $m$ is even. If $p=2$, we suppose $m \neq 1,3$, or 7 . Then $f_{(p)}$ is a homotopy equivalence.

From this we obtain the following

COROLlaRY 5.2. Let $f$ be a self-map of $\Omega^{k} S^{m+1}, k<m$, which induces an isomorphism on $H_{m+1-k}\left(\Omega^{k} S^{m+1} ; Z / p Z\right)$. If $p>2$, we suppose that $m$ is even, and if $p=2$, we suppose $m \neq 1,3$, or 7 . Then $f_{(p)}$ is a homotopy equivalence.

Received by the editors October 18, 1983.

1980 Mathematics Subject Classification. Primary 55P10, 55P35; Secondary 55P47.

${ }^{1}$ The first author is a Natural Sciences and Engineering Research Council of Canada postdoctoral fellow.

${ }^{2}$ The second author is partially supported by a National Science Foundation grant.

${ }^{3}$ The third author is a Natural Sciences and Engineering Research Council of Canada University research fellow. 
We also obtain versions of these results when $m=\infty$.

THEOREM 4.1. Let $f: \Omega_{0}^{\infty} S^{\infty} \rightarrow \Omega_{0}^{\infty} S^{\infty}$ be a loop map which induces an isomorphism on $H_{2 p-3}\left(\Omega_{0}^{\infty} S^{\infty} ; Z / p Z\right)$. Then $f_{(p)}$ is a homotopy equivalence.

COROLlARY 4.2. Let $f$ be a self-map of $\Omega^{\infty} S^{\infty} S^{t}, t \geqslant 2$, which induces an isomorphism on $H_{t}\left(\Omega^{\infty} S^{\infty} S^{t} ; Z / p Z\right)$. Then $f_{(p)}$ is a homotopy equivalence.

REMARK. We have simplified the statements of Theorems 5.1 and 4.1 for this introduction so that the reader may more easily appreciate that, in spite of the immense size of the homology of the space involved, a self-map satisfying surprisingly few hypotheses is forced to be a homotopy equivalence. The actual statements as presented in the text are slightly stronger. For example, if $p=2$, it suffices for $f$ to be an $H$-map which induces an isomorphism on $H_{1}(; Z / 2 Z)$.

This paper is rather lengthy and most of the work goes into the proof of Theorem 5.1. However, the proof of Theorem 4.1 is fairly short and straightforward. Readers interested only in the infinite loop case need only read the first half of $\S 1$ (to the end of Theorem 1.7) followed by the relatively short $\S \S 3$ and 4 (skipping Lemma 3.6).

In order to prove Theorem 5.1, we needed to calculate

$$
\text { Ann } P H^{*}\left(\Omega_{0}^{m+1} S^{m+1} ; Z / p Z\right) \text {, }
$$

the primitive elements which are annihilated by all Steenrod operations. This calculation, which should be of independent interest, is given in $\S 2$.

We have pointed out that Theorem 5.1 fails without the given restrictions on $m$. However, in each of these eliminated cases there exists an appropriate element $x \in H_{m}\left(\Omega_{0}^{m+1} S^{m+1}\right)$ such that, by adding an additional hypothesis which implies that $f_{*}(x)=x$, one can use the same arguments to get a similar theorem. We will leave these minor reformulations to the reader.

A space satisfying the property of Corollary 5.2 (that any self-map inducing an isomorphism on the lowest nonvanishing degree is a homotopy equivalence) was called atomic by Cohen, Moore and Neisendorfer [CMN]. We define a CW complex $X$ to be atomic at $p$ if $\tilde{H}_{i}(X ; Z / p Z)=0$ for $i<r, \tilde{H}_{r}(X ; Z / p Z) \neq 0$, and given any map $f: X \rightarrow X$ such that $f$ induces an isomorphism on $H_{r}(X ; Z / p Z)$, then $f_{(p)}$ is a homotopy equivalence. We define $X$ to be $H$-atomic at $p$ or loop-atomic at $p$ if $X$ is an $H$-space or loop space and the above condition is satisfied for any $f$ which is an $H$-map or loop map, respectively. Clearly, if $\Omega X$ is $H$-atomic or loop-atomic, then $X$ is atomic. If $X$ is atomic at $p$ and its least nonvanishing mod $p$ homology group has dimension one, then $X_{(p)}$ is indecomposable in the sense that it is not homotopy equivalent to a nontrivial product. Some results on indecomposability have previously been obtained by Wilkerson [W].

Cohen, Moore and Neisendorfer showed that most of the spaces occurring in their decompositions are atomic [CMN]. In their examples the homologies of the spaces involved were relatively small. Some other examples involving spaces with "small" homologies were considered by Selick [S2] and Cohen-Peterson-Selick [unpublished calculations in $\Omega^{3} S^{m+1}$ and $\Omega^{4} S^{m+1}$ ]. The proofs in all of these examples follow the same general pattern. One uses commutativity with the Steenrod operations and the 
coalgebra structure to show that the homological properties of any self-map are very rigidly restricted. This reduces the problem to showing that the induced map on homology is nonzero on a relatively small number of elements. One then applies ad hoc arguments (such as using the nonexistence of elements of Hopf invariant 1) to check these last few elements. Another quite different set of examples is the finite complex Grassmann manifolds, which are atomic at any prime $[\mathbf{G H}],[\mathbf{B H}],[\mathbf{H}]$.

When considering $\Omega^{k} S^{m+1}$, as $k$ grows one gains theoretical advantages since the resulting larger homology is more closely tied together by Steenrod operations so that the number of "special" elements for which one needs ad hoc arguments decreases. For example, the need to use Hopf invariant 1 in the proof of Cohen and Mahowald's theorem can be eliminated by looping. However, from the practical side, as the homology grows out of control it is no longer easy to comprehend the entire action of the Steenrod algebra, and so it becomes difficult to get one's hands on all the restrictions forced by commutativity with these operations. One has to wade through the wealth of information available and develop some machinery which will distill that information into a useful form. The reader will discover that some of our arguments are given in homology and others are given in cohomology after passing to primitives. Our guiding philosophy is to use cohomological arguments for the major work of reducing the problem to that of checking the small number of elements which are needed to get the inductions started, and to use homological arguments to do this checking.

This paper contains five sections. In $\S 1$ we describe the homology and cohomology of $\Omega_{0}^{m+1} S^{m+1}$ as modules over the Steenrod algebra. We begin by quoting material from [MCL]. In theory, the description contained there tells us everything there is to know about the structure of $H_{*}\left(\Omega_{0}^{m+1} S^{m+1} ; Z / p Z\right)$ as a Hopf algebra and as a Steenrod module, but we must piece the information together to obtain the formulae we need. $\$ 2$ contains the computation of Ann $P H^{*}\left(\Omega_{0}^{m+1} S^{m+1} ; Z / p Z\right)$. In $\S 3$ we introduce a self-map of $\Omega_{0}^{m+1} S^{m+1}$ and deduce those facts about its homological behaviour which are common to both the infinite loop case and the finite loop case. $\$ 4$ contains the proof of the main theorem in the infinite loop case and $\$ 5$ contains the proof in the finite loop case.

Note that although $H_{*}\left(\Omega_{0}^{m+1} S^{m+1} ; Z / p Z\right)$ is described in terms of Dyer-Lashof operations, our self-map, being only an $H$-map, need not commute with those operations. Thus the Dyer-Lashof operations serve only for bookkeeping. They provide names for the elements and help to describe the coalgebra structure and the action of the Steenrod operations.

The main technical results in which most of the work is done are Theorem $1.9, \S 2$, Lemma 4.4 (and preceding remarks), Theorems 5.3 and 5.4. The results of $\S 4$ are independent of $\$ 2$, which is nontrivial only in the finite loop case. The proofs of Theorems 5.3 and 5.4 are also independent of $\S 2$. Theorems 5.3 and 5.4 seem to be very close to the entire result in the finite loop case and, in fact, Theorem 5.3 by itself is sufficient to prove the main theorem if $m \equiv 0(p)$. Consequently, the results of $\$ 2$ are much more detailed than would be needed to finish the proof from Theorems 5.3 and 5.4 and so the finish of the proof of the theorem may seem like 
using the proverbial sledgehammer to drive in a nail. Perhaps the "ideal" approach would be to scrap Theorems 5.3 and 5.4 entirely and prove the theorem directly from the results of $\S 2$, but it was not clear to us how to do this.

As in [MCL] we will write the body of the paper as it would be for odd primes and put minor modifications necessary when $p=2$ in square brackets [ ]. When convenient $P_{*}^{r}$ will be used to denote $\mathrm{Sq}_{*}^{r}$ when the prime is 2 . We will be using the lower $Q$ notation for Dyer-Lashof operations. We find it convenient to let our lower $Q$ notation already contain the unit $v(q)$ in $Z / p Z$ which is usually introduced in defining the upper $Q$ notation from the lower $Q$ notion. (See [MCL, p. 7].) Thus our lower $Q$ notation differs by the unit $v(q)$ from that in [MCL]. One way to view our notation is to take the standard upper $Q$ notation and treat the equation $Q_{s(p-1)} y=$ $Q^{(s+|y|) / 2} y$ as the definition of our lower $Q$ notation when $p$ is odd.

In Self-maps of loop spaces, II, we will show that for many spaces $X, Q(X)=$ $\Omega^{\infty} S^{\infty} X$ is $H$-atomic at $p$ and hence $Q(S X)$ is atomic at $p$. For the prime 2 such spaces include $C P^{n}$ for $n<\infty$ and also $R P^{n}$ for $n \neq 1,3,7$, or $\infty$. However, $Q\left(C P^{\infty}\right)$ and $Q\left(R P^{\infty}\right)$ are not $H$-atomic at 2 by [J, B, Se, Sn], and Kahn-Priddy [KP]. We will also show how Theorem 4.1 gives a simple proof of the Kahn-Priddy Theorem.

We would like to thank F. R. Cohen very much for his many discussions with us while we worked on these results. He helped in our preliminary computations in checking the cases $\Omega^{3} S^{m+1}$ and $\Omega^{4} S^{m+1}$ and made a number of suggestions. More recently, he has been directly involved in studying $Q(X)$ and $\Omega^{m+1} S^{m+1} X$ and his name will appear on Self-maps of loop spaces, II.

Some of these results, in preliminary form, have appeared in [P and S1].

1. Homology and cohomology of $\Omega^{m+1} S^{m+1}$. In this section we describe $P H^{*}\left(\Omega_{0}^{m+1} S^{m+1} ; Z / p Z\right)$ as an $A$-module where $A$ denotes the $(\bmod p)$ Steenrod Algebra and $\Omega_{0}^{m+1} S^{m+1}$ denotes the component of the basepoint of $\Omega^{m+1} S^{m+1}$. In particular, we will obtain explicit formulae for some of the action of the Milnor primitives of the Steenrod algebra on $P H^{*}\left(\Omega_{0}^{m+1} S^{m+1} ; Z / p Z\right)$. This action turns out to be fairly straightforward and contains most of the information that we will need. We will also describe the epimorphism

$$
P H^{*}\left(\Omega_{0}^{\infty} S^{\infty} ; Z / p Z\right) \rightarrow P H^{*}\left(\Omega_{0}^{m+1} S^{m+1} ; Z / p Z\right)
$$

induced by the canonical map from $\Omega^{m+1} S^{m+1}$ to $\Omega^{\infty} S^{\infty}$. If $p>2$ and $m<\infty$ we will assume $m$ is even. Let

$$
n=\left\{\begin{array}{ll}
m / 2 & \text { if } p>2 \\
m & \text { if } p=2
\end{array}\right\} .
$$

$H_{*}\left(\Omega^{m+1} S^{m+1} ; Z / p Z\right)$ is described in [MCL] in terms of Dyer-Lashof operations. It will be convenient to use the lower $Q$ notation for the Dyer-Lashof operations. That is, if $p>2, Q_{s(p-1)} y=Q^{(s+|y|) / 2} y$ provided $s+|y|$ is even, and if $p=2, Q_{s} y=Q^{s+|y|} y$ for every $s$. Note that this differs by a unit in $Z / p Z$ from the notation in [MCL]. (See the Introduction.) $Q_{0}$ is the $p$ th power operation. Since we have assumed $m$ to be even when $p>2$, the Browder operations are trivial, so it 
should cause no confusion if we denote the "top" operation by $Q_{m(p-1)}$. If $I=\left(i_{1}(p-1), \ldots, i_{k}(p-1)\right)$ and $J=\left(\varepsilon_{1}, \ldots, \varepsilon_{k}\right)$ where $\varepsilon_{j}=0$ or 1 we let $Q_{I, J}$ denote the composition of operations

$$
\beta^{\varepsilon_{1}} Q_{i_{1}(p-1)} \beta^{\varepsilon_{2}} Q_{i_{2}(p-1)} \cdots \beta^{\varepsilon_{k}} Q_{i_{k}(p-1)} .
$$

It will be admissible provided $0 \leqslant i_{j} \leqslant i_{j+1}-\varepsilon_{j+1}$. Let $l(I, J)=k$. If $p=2$ or if all entries of $J$ are zero, we will simply write $Q_{I}$. Note that the notation is somewhat redundant in that all of the entries of $J$ except for the first are actually determined by $I$. Namely, $\varepsilon_{j+1}$ is the congruence class of $i_{j+1}-i_{j}$ modulo 2 . The Adem relations in lower notation are

$$
\begin{aligned}
& Q_{a(p-1)} Q_{b(p-1)} \\
&=\sum_{j}(-1)^{(a+(p-1) b) / 2+j}\left(\begin{array}{c}
(2 j-b)\left(\frac{p-1}{2}\right)-1 \\
a / 2-j-1
\end{array}\right) Q_{(a+p b-2 p j)(p-1)} Q_{2 j(p-1)} \\
& {\left[\begin{array}{c}
\sum_{j}\left(\begin{array}{c}
j-b-1 \\
a-j-1
\end{array}\right) Q_{a+2 b-2 j} Q_{j}
\end{array}\right], }
\end{aligned}
$$

and if $p>2$,

$$
\begin{aligned}
& Q_{a(p-1)} \beta Q_{b(p-1)} \\
& \quad=\sum_{j}(-1)^{(a-1+(p-1) b) / 2+j}\left(\begin{array}{c}
(2 j-b)\left(\frac{p-1}{2}\right) \\
(a-1) / 2-j
\end{array}\right) \beta Q_{(a-1+p b-2 p j)(p-1)} Q_{2 j(p-1)} \\
& \quad+\sum_{j}(-1)^{a-1+(p-1) b / 2+j}\left(\begin{array}{c}
(2 j-b)\left(\frac{p-1}{2}\right)-1 \\
(a-1) / 2-j
\end{array}\right) Q_{(a+p b-2 p j)(p-1)} \beta Q_{2 j(p-1)} .
\end{aligned}
$$

In $H_{0}\left(\Omega^{m+1} S^{m+1} ; Z / p Z\right)$ let [1] denote the image of a generator of $\tilde{H}_{0}\left(S^{0} ; Z / p Z\right)$ under $S^{0} \rightarrow \Omega^{m+1} S^{m+1}$ and let $[r]=[1]^{r}$ for $r \in Z$. According to [MCL], $\quad H_{*}\left(\Omega_{0}^{m+1} S^{m+1} ; Z / p Z\right)$ is the free commutative graded algebra on $\left\{Q_{I, J}[1] *\left[-p^{l(I, J)}\right]\right\}$ where $(I, J)$ runs through the admissible sequences with $i_{1}>0$ and $i_{l(I, J)} \leqslant m(p-1)$ and $*$ denotes Pontryagin multiplication.

For a space $X$ such that $H_{*}(X ; Z / p Z)$ has finite type, the hom-duals of

$$
\begin{gathered}
P^{r}: H^{q}(X ; Z / p Z) \rightarrow H^{q+2 r(p-1)}(X ; Z / p Z) \\
{\left[\mathrm{Sq}^{r}: H^{q}(X ; Z / 2 Z) \rightarrow H^{q+r}(X ; Z / 2 Z)\right]}
\end{gathered}
$$

are denoted

$$
\begin{gathered}
P_{*}^{r}: H_{s}(X ; Z / p Z) \rightarrow H_{s-2 r(p-1)}(X ; Z / p Z) \\
{\left[\mathrm{Sq}_{*}^{r}: H_{s}(X ; Z / 2 Z) \rightarrow H_{s-r}(X ; Z / 2 Z)\right] .}
\end{gathered}
$$


They turn $H_{*}(X ; Z / p Z)$ into an $A_{*}$-module, where $A_{*}$ denotes the opposite algebra of the Steenrod Algebra A. The Nishida relations in lower $Q$ notation are

$$
\begin{aligned}
P_{*}^{r} Q_{s(p-1)} x=\sum_{j}(-1)^{r+j}\left(\begin{array}{r}
(s-2 r+|x|)\left(\frac{p-1}{2}\right) \\
r-p j
\end{array}\right) Q_{(s-2 r+2 p j)(p-1)} P_{*}^{j} x & {\left[\begin{array}{c}
\sum_{j}\left(\begin{array}{c}
s+|x|-r \\
r-2 j
\end{array}\right) Q_{s-r+2 j} \mathrm{Sq}_{*}^{j} x
\end{array}\right], }
\end{aligned}
$$

and if $p>2$,

$$
\begin{gathered}
P_{*}^{r} \beta Q_{s(p-1)} x=\sum_{j}(-1)^{r+j}\left(\begin{array}{c}
(s-2 r+|x|)\left(\frac{p-1}{2}\right)-1 \\
r-p j
\end{array}\right) \beta Q_{(s-2 r+2 p j)(p-1)} P_{*}^{j} x \\
+\sum_{j}(-1)^{r+j}\left(\begin{array}{c}
(s-2 r+|x|)\left(\frac{p-1}{2}\right)-1 \\
r-p j-1
\end{array}\right) Q_{(s-2 r+2 p j+1)(p+1)} P_{*}^{j} \beta x .
\end{gathered}
$$

Let $R$ denote the $(\bmod p)$ Dyer-Lashof Algebra. Within $R$ the notation $Q_{I, J}$ shall be interpreted as the element which in upper notation corresponds to $Q_{I, J}$ when evaluated on a class in degree 0 . Let

$$
\begin{aligned}
R_{n}= & \left.\left\langle Q_{I, J} \in R\right| i_{j} \leqslant 2 n \text { for all } j \text { where } I=\left(i_{1}(p-1), \ldots, i_{k}(p-1)\right)\right\rangle \\
& \left.\cdot\left[\left\langle Q_{I} \in R\right| i_{j} \leqslant n \text { for all } j \text { where } I=\left(i_{1}, \ldots, i_{k}\right)\right\rangle\right] .
\end{aligned}
$$

Define $\Phi: R_{n} \rightarrow H_{*}\left(\Omega_{0}^{m+1} S^{m+1} ; Z / p Z\right)$ by $\Phi\left(Q_{I, J}\right)=Q_{I, J}[1] *\left[-p^{l(I, J)}\right]$. If the Nishida relations are used to define an $A_{*}$-module structure on $R_{n}$, then $\Phi$ is an $A_{*}$-module homomorphism. Let $Q_{0} R_{n}=\left\langle Q_{I, J} \in R_{n} \mid i_{1}=0\right\rangle$. The composite

$$
R_{n} \stackrel{\oplus}{\rightarrow} H_{*}\left(\Omega_{0}^{m+1} S^{m+1} ; Z / p Z\right) \rightarrow Q H_{*}\left(\Omega_{0}^{m+1} S^{m+1} ; Z / p Z\right)
$$

is an epimorphism with kernel $Q_{0} R_{n}$, and so we have an $A_{*}$-module isomorphism

$$
Q H_{*}\left(\Omega_{0}^{m+1} S^{m+1} ; Z / p Z\right) \cong R_{n} / Q_{0} R_{n} .
$$

Let

$$
R_{n}[k]=\left\langle Q_{I, J} \in R_{n} \mid l(I, J)=k\right\rangle
$$

and let

$$
Q_{0} R_{n}[k]=\left\langle Q_{I, J} \in R_{n}[k] \mid i_{1}=0\right\rangle .
$$

$R_{n}[k]$ is an $A_{*}$-coalgebra and $Q_{0} R_{n}[k]$ is an $A_{*}$-subcoalgebra. We have $R_{n} / Q_{0} R_{n}$ $=\oplus_{k} R_{n}[k] / Q_{0} R_{n}[k]$ so that the dualizing (1) gives an $A$-module isomorphism

$$
\begin{aligned}
P H^{*}\left(\Omega_{0}^{m+1} S^{m+1} ; Z / p Z\right) & \cong \prod_{k}\left(R_{n}[k] / Q_{0} R_{n}[k]\right)^{*} \\
& \cong \bigoplus_{k}\left(R_{n}[k] / Q_{0} R_{n}[k]\right)^{*}
\end{aligned}
$$

since $P H^{*}\left(\Omega_{0}^{m+1} S^{m+1} ; Z / p Z\right)$ has finite type. 
REMARK. For each $k,\left(R_{n}[k] / Q_{0} R_{n}[k]\right)^{*}$ has a multiplicative structure as described below but this structure is not related under the above isomorphism to any natural multiplicative structure in $P H^{*}\left(\Omega_{0}^{m+1} S^{m+1} ; Z / p Z\right)$. In particular, it is not related to the multiplication in $H^{*}\left(\Omega_{0}^{m+1} S^{m+1} ; Z / p Z\right)$ under which the product of primitives is of course not primitive in general. Thus, looking ahead to $\S 3$, the map $f^{*}[k]$ described there will be an $A$-module map only and need not preserve multiplicative structure.

In order to describe $\left(R[k] / Q_{0} R[k]\right)$ as an $A$-module let

$$
a_{j k}=Q_{(\underbrace{0, \ldots, 0,2(p-1), \ldots, 2(p-1))}_{k-j}} \underbrace{}_{j}\left[Q_{(\underbrace{(0, \ldots, 0,0,1, \ldots, 1)^{*}}_{k-j}}\right],
$$

$j \leqslant k$, and if $p>2$,

$$
\begin{aligned}
& \tau_{j k}=Q_{k-j}^{(\underbrace{p-1, \ldots, p-1}} \underbrace{2(p-1), \ldots, 2(p-1))}_{j},(0, \ldots, 0,1,0, \ldots, 0)^{*}, \quad j \leqslant k,
\end{aligned}
$$

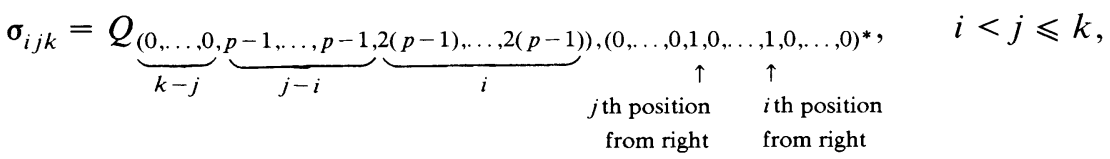

in the dual basis to the basis of admissible sequences for $R_{n}[k]$. Thus, $\left|a_{j k}\right|=$ $2\left(p^{k}-p^{k-j}\right)\left[2^{k}-2^{k-j}\right], \quad\left|\tau_{j k}\right|=2\left(p^{k}-p^{k-j}\right)-1, \quad\left|\sigma_{i j k}\right|=2\left(p^{k}-p^{k-i}-p^{k-j}\right)$. By convention, $\sigma_{i j k}=0$ if $i \geqslant j$. When dealing with a fixed $k$ we drop the last subscript.

TheOREM 1.1 (MAY $p>2$, MADSEN $p=2$ ). As an A-algebra $R[k]^{*} \cong$ free commutative graded algebra on $\left\{a_{j}, \tau_{j}, \sigma_{i j}\right\}\left[\left\{a_{j}\right\}\right]$ modulo the relations
(i) $\tau_{i} \tau_{j}=a_{k} \sigma_{i j}$,
(ii) $\sigma_{i j} \tau_{s}=\tau_{i} \tau_{j} \tau_{s} / a_{k}$,
(iii) $\sigma_{i j} \sigma_{s t}=\tau_{i} \tau_{j} \tau_{s} \tau_{t} / a_{k}^{2}$,

with

$$
P^{p^{t}} a_{j}=\left\{\begin{array}{ll}
-\delta_{j}^{k-t-1} a_{j+1}, & t<k-1 \\
a_{1} a_{j}, & t=k-1 \\
0, & t>k-1
\end{array}\right\},
$$

and if $p>2$,

$$
\begin{gathered}
P^{p^{t}} \tau_{j}=\left\{\begin{array}{ll}
-\delta_{j}^{k-t-1} \tau_{j+1}, & t<k-1 \\
-a_{1} \tau_{j}+a_{j} \tau_{1}, & t=k-1 \\
0, & t>k-1
\end{array}\right\}, \\
P^{p^{t} \sigma_{i j}}=\left\{\begin{array}{ll}
-\delta_{i}^{k-t-1} \sigma_{i+1, j}-\delta_{j}^{k-t-1} \sigma_{i, j+1}, & t<k-1 \\
a_{1} \sigma_{i j}+a_{i} \sigma_{1 j}-a_{j} \sigma_{1 i}, & t=k-1 \\
0, & t>k-1
\end{array}\right\}, \\
\beta \tau_{k}=a_{k}, \quad \beta \sigma_{i k}=-\tau_{i}, \quad \beta \tau_{j}=\beta \sigma_{i j}=0(j<k), \quad \beta a_{j}=0 .
\end{gathered}
$$


Proof. See May [MCL, §I.3] and Madsen [Ma, §3].

As an algebra $R[k]^{*} \cong P[k] \otimes M[k]$, where $P[k]=Z / p Z\left[a_{1}, \ldots, a_{k}\right]$ is the subalgebra of $R[k]^{*}$ generated by $\left\{a_{j}\right\}$ and $M[k]$ is the subalgebra generated by $\left\{\tau_{j}, \sigma_{i j}\right\}$. Observe that $P[k]$ is closed under the Steenrod operations and so forms an $A$ subalgebra. Let $P=\oplus_{k} P[k]$.

Let $P^{\Delta_{s}}, s \geqslant 1$, and $Q^{\Delta_{s}}, s \geqslant 0$, denote the elements of $A$ defined inductively by Milnor as $P^{\Delta_{1}}=P^{1}, P^{\Delta_{s}}=\left[P^{p^{s-1}}, P^{\Delta_{s-1}}\right]$ and $Q^{\Delta_{0}}=\beta, Q^{\Delta_{s}}=\left[P^{p^{s-1}}, Q^{\Delta_{s-1}}\right]$. Using the inductive definition above and the fact that $P^{\Delta_{s}}$ and $Q^{\Delta_{s}}$ are primitive and thus act as derivations, it is easy to verify that the actions of $P^{\Delta_{s}}$ and $Q^{\Delta_{s}}$ for $s \leqslant k$ are given by the following

THEOREM 1.2.

$$
\begin{aligned}
& P^{\Delta_{s}} a_{j}=-\delta_{j}^{k-s} a_{k}, \quad s<k, \\
& P^{\Delta_{k}} a_{j}=a_{j} a_{k},
\end{aligned}
$$

and if $p>2$,

$$
\begin{aligned}
Q^{\Delta_{s}} a_{j} & =0, \\
P^{\Delta_{s}} \tau_{j} & =-\delta_{j}^{k-s} \tau_{k}, \quad s<k, \\
P^{\Delta_{k}} \tau_{j} & =a_{k} \tau_{j}+a_{j} \tau_{k}, \\
Q^{\Delta_{s}} \tau_{j} & =-\delta_{j}^{k-s} a_{k}, \quad s<k, \\
Q^{\Delta_{k}} \tau_{j} & =a_{j} a_{k}, \\
P^{\Delta_{s}} \sigma_{i j} & =\delta_{i}^{k-s} \sigma_{j k}-\delta_{j}^{k-s} \sigma_{i k}, \quad s<k, \\
P^{\Delta_{k}} \sigma_{i j} & =a_{j} \sigma_{i k}+a_{k} \sigma_{i j}-a_{i} \sigma_{j k}, \\
Q^{\Delta_{s}} \sigma_{i j} & =\delta_{j}^{k-s} \tau_{i}-\delta_{i}^{k-s} \tau_{j}, \quad s<k, \\
Q^{\Delta_{k}} \sigma_{i j} & =a_{i} \tau_{j}-a_{j} \tau_{i} . \quad \square
\end{aligned}
$$

Corollary 1.3. Let $a=a_{1}^{r_{1}} \cdots a_{k}^{r_{k}}$ be a monomial in $Z / p Z\left[a_{1}, \ldots, a_{k}\right]$ and let $r=\sum r_{i}$. Then

(i) $P^{\Delta_{s}} a=(-1)^{r_{k-s}} r_{k-s} a\left(a_{k} / a_{k-s}\right), 1 \leqslant s \leqslant k-1$,

(ii) $P^{\Delta_{k}} a=r a a_{k}$.

COROllary 1.4. $y \in Z / p Z\left[a_{1}, \ldots, a_{k}\right]$. Then $y$ is a pth power if and only if $y \in \bigcap_{j=1}^{k} \operatorname{Ker} P^{\Delta_{j}}$ if and only if $y \in \bigcap_{j=1}^{\infty} \operatorname{Ker} P^{\Delta_{j}}$.

Proof. $\{p$ th powers $\}=\bigcap_{j=1}^{k} \operatorname{Ker} P^{\Delta_{j}}$ is immediate from Corollary 1.3. However, since $P^{\Delta_{j}}$ is primitive for all $j$,

$$
\{p \text { th powers }\} \subset \bigcap_{j=1}^{\infty} \operatorname{Ker} P^{\Delta_{j}} \subset \bigcap_{j=1}^{k} \operatorname{Ker} P^{\Delta_{j}} .
$$


THEOREM 1.5. $P[k]=\bigcap_{s=0}^{k-1} \operatorname{Ker} Q^{\Delta_{s}}$.

Proof. $P[k] \subset \bigcap_{s=0}^{k-1} \operatorname{Ker} Q^{\Delta_{s}}$ is immediate from Theorem 1.2. Conversely, given a nonzero $y$ in $R[k]^{*}$, write $y$ as a sum of a minimal number of monomials in $\left\{a_{j}, \tau_{j}, \sigma_{i j}\right\}$. By inspection, if $\tau_{i}$ or $\sigma_{i j}$ occurs in any of these monomials, then $Q^{\Delta_{k-i}} y \neq 0$ and so $\bigcap_{s=0}^{k-1} \operatorname{Ker} Q^{\Delta_{s}} \subset P[k]$.

The above description of $R[k]^{*}$ gives an obvious basis of monomials in $\left\{a_{j}, \tau_{j}, \sigma_{i j}\right\}$. Define a set function from this basis to (monotonically increasing sequences in $\left.\left(Z^{+}\right)^{k}\right) \times(Z / 2 Z)^{k}$ by

$$
\begin{aligned}
& \psi\left(a_{j}\right)=(\underbrace{0, \ldots, 0}_{k-j}, \underbrace{2(p-1), \ldots, 2(p-1)}_{j}),(0, \ldots, 0) \quad[(\underbrace{0, \ldots, 0}_{k-j}, \underbrace{1, \ldots, 1}_{j})], \\
& \psi\left(\tau_{j}\right)=\underbrace{(p-1, \ldots, p-1}_{k-j}, \underbrace{2(p-1), \ldots, 2(p-1))}_{j},(0, \ldots, 0,1,0, \ldots, 0), \\
& \psi\left(\sigma_{i j}\right)=(\underbrace{0, \ldots, 0}_{k-j}, \underbrace{p-1, \ldots, p-1}_{j-i}, \underbrace{2(p-1), \ldots, 2(p-1)}_{i}),
\end{aligned}
$$

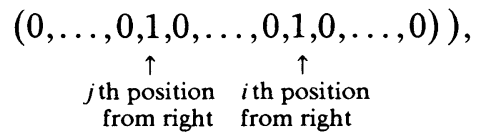

and $\psi(x y)=\psi(x)+\psi(y)$. We order the monotonically increasing sequences lexicographically from the left. The correspondence $x \rightarrow Q_{\psi(x)}$ is a set bijection from the basis of monomials for $R[k]^{*}$ to a basis for $R[k]$.

Lemma 1.6 (MAY). $\left\langle x, Q_{\psi(x)}\right\rangle=1$. Conversely, if $\left\langle y, Q_{\psi(x)}\right\rangle \neq 0$, then $\psi(y) \leqslant$ $\psi(x)$.

Proof. See [MCL, p. 30].

THEOREM 1.7. $\left(R[k] / Q_{0} R[k]\right)^{*} \cong I[k]$ where $I[k]$ is the ideal in $R[k]^{*}$ generated by $a_{k},\left\{\tau_{i}\right\}$ and $\left\{\sigma_{i k}\right\}$.

Proof. $\left\langle I[k], Q_{0} R[k]\right\rangle=0$ is immediate from Lemma 1.6. Thus $I[k] \subset$ $\left(R[k] / Q_{0} R[k]\right)^{*}$. Further, $\psi$ sets up a bijection from a basis of $I[k]$ to a basis of $R[k] / Q_{0} R[k]$ and thus we have equality.

Let $j_{n}$ be the inclusion $R_{n}[k] \nrightarrow R[k]$ so that $j_{n}^{*}$ maps $R[k]^{*}$ onto $R_{n}[k]^{*}$. Thus $P H^{*}\left(\Omega_{0}^{m+1} S^{m+1} ; Z / p Z\right) \cong \oplus_{k} j_{n}^{*}(I[k])$. Lemma 1.6 and a counting argument using the bijection $\psi$ show that the images under $j_{n}^{*}$ of those monomials in the basis of $P[k] \otimes M[k]$, which are the product of at most $n$ factors, form a basis for $R_{n}[k]^{*}$. We will abuse notation and write elements in $P[k] \otimes M[k]$ for their images under $j_{n}^{*}$. The monomials in $P[k] \otimes M[k]$ which are the product of at most $n$ factors will be called admissible in $R_{n}[k]^{*}$. For $x \in j_{n}^{*}(P[k])$, there is precisely one admissible representative for $x$, and we will let $d(x)$ denote the polynomial degree of this admissible representative. 
CoROLlaRY 1.8. In $R_{n}[k]^{*}, j_{n}^{*}(P[k])=\bigcap_{s=0}^{k-1} \operatorname{Ker} Q^{\Delta_{s}}=\bigcap_{s=0}^{\infty} \operatorname{Ker} Q^{\Delta_{s}}$.

Proof. The first equality follows immediately from Theorem 1.5 and the fact that, for $s \leqslant k-1$, application of $Q^{\Delta_{s}}$ does not change the number of factors in a monomial. However, $j_{n}^{*}(P[k]) \subset \bigcap_{s=0}^{\infty} \operatorname{Ker} Q^{\Delta_{s}} \subset \bigcap_{s=0}^{k-1} \operatorname{Ker} Q^{\Delta_{s}}$ is also clear from Theorem 1.2.

We next describe how to write polynomials in $P$ of degree greater than $n$ admissibly in $R_{n}[k]^{*}$. In other words, given a polynomial $x \in P[k]$ with $d(x)>n$, we describe how to find the unique polynomial $y \in P[k]$ with $d(y) \leqslant n$ such that $j_{n}^{*} x=j_{n}^{*} y$. This description and its properties will be used in the proof of Theorem 5.4 .

In addition to the basis of monomials in $\left\{a_{j}, \tau_{j}, \sigma_{i j}\right\}$ there is also in $R_{n}[k]^{*}$ the dual basis to the basis of admissible monomials spanning $R_{n}[k]$. Given $x \in P[k]$, possibly written inadmissibly, we first describe how to write $x$ in the dual basis. Let $a$ be a monomial in $P[k]$. We want to find all sequences $J$ such that $\left\langle a, Q_{2(p-1) J}\right\rangle \neq 0$ $\left[\left\langle a, Q_{J}\right\rangle \neq 0\right]$. Let $r=d(a)$ and write $a=a_{s_{1}} \cdot a_{s_{2}} \cdot \cdots a_{s_{r}}$.

$$
\begin{aligned}
\left\langle a, Q_{2(p-1) J}\right\rangle[ & \left.\left\langle a, Q_{J}\right\rangle\right] \\
& =\left\langle a_{s_{1}} \otimes \cdots \otimes a_{s_{r}}, \psi^{r} Q_{2(p-1) J}\right\rangle\left[\left\langle a_{s_{1}} \otimes \cdots \otimes a_{s_{r}}, \psi^{r} Q_{J}\right\rangle\right] \\
& =\text { number of terms of form } Q_{\psi\left(a_{s_{1}}\right)} \otimes Q_{\psi\left(a_{s_{2}}\right)} \otimes \cdots \otimes Q_{\psi\left(a_{s_{r}}\right)} \\
& \operatorname{in} \psi^{r} Q_{2(p-1) J}\left[\psi^{r} Q_{j}\right] \\
& =\text { number of sequences } J_{1}, \ldots, J_{r} \text { such that } Q_{2(p-1) J_{i}}=Q_{\psi\left(a_{s_{i}}\right)}
\end{aligned}
$$

$$
\left[Q_{J_{i}}=Q_{\psi\left(a_{s_{i}}\right)}\right] \text { under the Adem relations and } \sum J_{i}=J \text {. }
$$

By inspection, $Q_{2(p-1)} Q_{2(p-1)}\left[Q_{1} Q_{1}\right]$ appears in no Adem relation, so the only Adem relation which can be used to get $Q_{\psi\left(a_{s_{i}}\right)}$ from $Q_{2(p-1) J_{i}}\left[Q_{J_{i}}\right]$ is

$$
Q_{2 p^{t}(p-1)} Q_{0}=Q_{0} Q_{2 p^{t-1}(p-1)} \quad\left[Q_{2^{t}} Q_{0}=Q_{0} Q_{2^{t-1}}\right]
$$

A convenient method of working backwards from $\psi\left(a_{s_{1}}\right), \ldots, \psi\left(a_{s_{r}}\right)$ to find the possible $J$ 's is as follows: Form the $r \times k$ matrix whose $i$ th row is $(1 / 2(p-1)) \psi\left(a_{s_{i}}\right)$ [ $\psi\left(a_{s_{i}}\right)$. Consider the set $\mathscr{S}(a)$ of all $r \times k$ matrices $M$ whose $i$ th row $M_{i}$ has the property that $Q_{2(p-1) M_{i}}=Q_{\psi\left(a_{i}\right)}\left[Q_{M_{i}}=Q_{\psi\left(a_{i}\right)}\right]$ under the Adem relation above.

For each matrix $M \in \mathscr{S}(a)$, let $S(M)$ be the sequence formed by summing its columns. We will use the phrase " $J$ is admissible" to mean that $Q_{2(p-1) J}\left[Q_{J}\right] \in R_{n}$ is admissible. We have $\left\langle a, Q_{2(p-1) J}\right\rangle\left[\left\langle a, Q_{J}\right\rangle\right]=$ number of such matrices for which $S(M)=J$. Thus,

$$
\begin{aligned}
a= & \sum_{\{J \mid J \text { is admissible }\}}\left\langle a, Q_{2(p-1) J}\right\rangle Q_{2(p-1) J}^{*} \\
& {\left[\sum_{\{J \mid J \text { is admissible }\}}\left\langle a, Q_{J}\right\rangle Q_{J}^{*}\right] } \\
= & \sum_{\{M \in \mathscr{S}(a) \mid S(M) \text { is admissible }\}} Q_{2(p-1) S(M)}^{*} \\
& {\left[\sum_{\{M \in \mathscr{S}(a) \mid S(M) \text { is admissible }\}} Q_{S(M)}^{*}\right] . }
\end{aligned}
$$


To compute $Q_{2(p-1)}^{*}\left[Q_{J}^{*}\right]$ in terms of the polynomial basis use (2) to compute

$$
\begin{gathered}
\psi^{-1}(2(p-1) J)=* Q_{2(p-1) J}+\sum u_{i} Q_{2(p-1) J_{i}}^{*} \\
{\left[\psi^{-1}(J)=Q_{J}^{*}+\sum Q_{J_{i}}^{*}\right],}
\end{gathered}
$$

and so $Q_{2(p-1) J}^{*}=\psi^{-1}(2(p-1) J)-\sum u_{i} Q_{2(p-1) J_{i}}^{*}\left[Q_{J}^{*}=\psi^{-1}(J)+\sum Q_{J_{i}}^{*}\right]$. We can then use (2) again to get a similar expression for each $Q_{2(p-1) J_{i}}^{*}\left[Q_{J_{i}}^{*}\right]$. Since $J<J_{i}$ for all $i$ by Lemma 1.6, the procedure eventually terminates.

EXAmple. Let $p=2, k=3, n=11$. We will compute the admissible form of $a_{1}^{9} a_{2}^{2} a_{3}$. We begin with the $12 \times 3$ matrix

$$
\begin{aligned}
8 & \left\{\left(\begin{array}{lll}
0 & 0 & 1 \\
0 & 0 & 1 \\
& \vdots & \\
0 & 0 & 1 \\
0 & 1 & 1 \\
0 & 1 & 1 \\
1 & 1 & 1
\end{array}\right) .\right. \\
& \{.
\end{aligned}
$$

Since we are working modulo 2 , in computing $\mathscr{S}\left(a_{1}^{9} a_{2}^{2} a_{3}\right)$ it suffices to consider only matrices $M$ whose braced rows are identical, since for other $M, S(M)$ will occur an even number of times by symmetry. With this in mind, we get the following matrices:

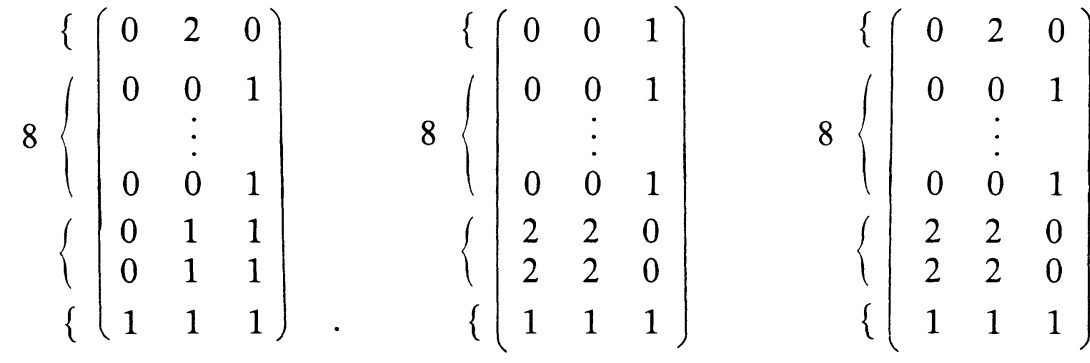

$$
\begin{aligned}
& S(M)=\left(\begin{array}{lll}
1 & 5 & 11
\end{array}\right) \quad\left(\begin{array}{lll}
5 & 5 & 10
\end{array}\right) \quad\left(\begin{array}{lll}
5 & 7 & 9
\end{array}\right) \text {. }
\end{aligned}
$$

So $a_{1}^{9} a_{2}^{2} a_{3}=Q_{(1,5,11)}{ }^{*}+Q_{(5,5,10)}{ }^{*}+Q_{(5,7,9)}^{*}$.

$\psi^{-1}(1,5,11)=a_{1}^{6} a_{2}^{4} a_{3}$, so we consider

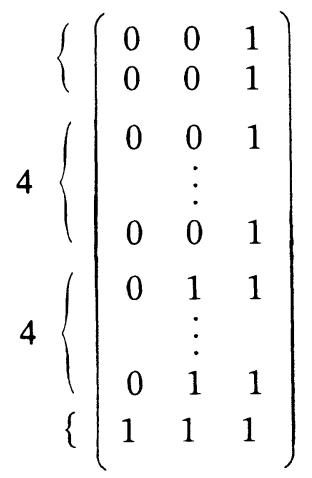


which leads to the matrices

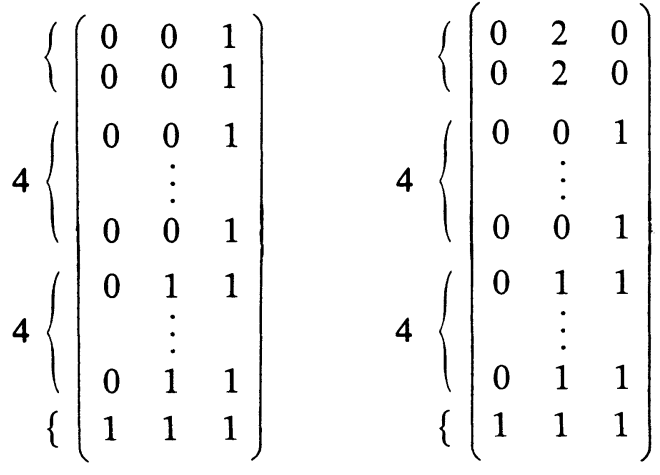

$$
\begin{aligned}
& S(M)=\left(\begin{array}{lll}
1 & 5 & 11
\end{array}\right) \quad\left(\begin{array}{lll}
1 & 9 & 9
\end{array}\right) \text {. }
\end{aligned}
$$

So $a_{1}^{6} a_{2}^{4} a_{3}=Q_{(1,5,11)}{ }^{*}+Q_{(1,9,9)}{ }^{*} \cdot \psi^{-1}(5,5,10)=a_{1}^{5} a_{3}^{5}$, so consider

$$
\begin{aligned}
4 & \left\{\left(\begin{array}{lll}
0 & 0 & 1 \\
0 & 0 & 1 \\
& \vdots & \\
0 & 0 & 1 \\
1 & 1 & 1 \\
1 & 1 & 1 \\
4 & \vdots & \\
1 & 1 & 1
\end{array}\right)\right.
\end{aligned}
$$

leading to

$$
\begin{aligned}
& \begin{array}{l}
\left\{\left\{\left(\begin{array}{lll}
0 & 0 & 1 \\
0 & 0 & 1 \\
& \vdots & \\
0 & 0 & 1 \\
1 & 1 & 1 \\
1 & 1 & 1 \\
4 & \vdots & \\
1 & 1 & 1
\end{array}\right)\right.\right.
\end{array} \\
& S(M)=\left(\begin{array}{lll}
5 & 5 & 10
\end{array}\right) \\
& \begin{array}{c}
\left\{\left\{\left(\begin{array}{lll}
0 & 2 & 0 \\
0 & 0 & 1 \\
& \vdots & \\
0 & 0 & 1 \\
1 & 1 & 1 \\
1 & 1 & 1 \\
4 & \vdots & \\
1 & 1 & 1
\end{array}\right)\right.\right.
\end{array} \\
& \text { (l5 } 7 \text { ( } 9 \text { ). }
\end{aligned}
$$

Thus $a_{1}^{5} a_{3}^{5}=Q_{(5,5,10)}^{*}+Q_{(5,7,9)}^{*}$. Finally, $a_{2}^{8} a_{3}=Q_{(1,9,9)}^{*}$ is verified by the same method, so we conclude $a_{1}^{9} a_{2}^{2} a_{3}=a_{1}^{6} a_{2}^{4} a_{3}+a_{2}^{8} a_{3}+a_{1}^{5} a_{3}^{4}$.

The following properties of this description of $j_{n}^{*}$ will be used in the proof of Theorem 5.4.

LEMMA 1.9. Let $x=a_{1}^{r_{1}} \cdots a_{k}^{r_{k}} \in P[k]$. Suppose that $d(x)=n, r_{i} \equiv 0(p)$ for $1 \leqslant i<k$, and that $r_{1} \geqslant p$. Then in $R_{n}[k]^{*}, a_{1} x \equiv x\left(a_{2} / a_{1}\right)^{p}$ modulo polynomials of degree less than $n+2-p\left(\right.$ where $a_{2}=0$ by convention if $\left.k=1\right)$. 
Proof. Let

$$
A=p\left\{\left(\begin{array}{cccc}
0 & \cdots & 0 & 1 \\
0 & \cdots & 0 & 1 \\
& \vdots & & \\
0 & \cdots & 0 & 1
\end{array}\right)\right.
$$

be the starting matrix for $a_{1} x$. Then $\mathscr{S}\left(a_{1} x\right)$ contains the matrix

$$
B=p\left\{\left(\begin{array}{ccccc}
0 & \cdots & 0 & p & 0 \\
0 & \cdots & 0 & 0 & 1 \\
& & \vdots & & \\
0 & \cdots & 0 & 0 & 1
\end{array}\right)\right.
$$

and $\mathscr{S}\left(x\left(a_{2} / a_{1}\right)^{p}\right)$ contains the matrix

$$
C=\left(\begin{array}{lllll}
0 & \cdots & 0 & p & p \\
& & * & &
\end{array}\right) .
$$

Note that $S(B)=S(C)$. It is clear that if $J$ is admissible and $j_{k}<r$ then $d\left(Q_{2(p-1) J}^{*}\right)$ $<r\left[d\left(Q_{J}^{*}\right)<r\right]$ when written in the polynomial basis. Thus, in computing $\mathscr{S}\left(a_{1} x\right)$ and $\mathscr{S}\left(x\left(a_{2} / a_{1}\right)^{p}\right)$ it suffices to consider only matrices $M$ whose last column sums to at least $n+2-p$. Since $r_{i} \equiv 0(p)$ when $i<k$ and we are working modulo $p$, in computing $\mathscr{S}\left(a_{1} x\right)$ and $\mathscr{S}\left(x\left(a_{2} / a_{1}\right)^{p}\right)$ it suffices to divide the rows other than the first row and the last $r_{k}$ rows into groups of $p$ identical rows and consider only matrices in which the groups of $p$ rows remain identical, since for other $M$ the number of times $S(M)$ occurs will be divisible by $p$ by symmetry. Since no Adem relations are possible on the last $r_{k}$ rows and we are interested only in matrices whose last column sums to at least $n+2-p$, in computing $\mathscr{S}\left(a_{1} x\right)$ it suffices to consider only matrices whose last column is identical to that of $B$, and similarly in computing $\mathscr{S}\left(x\left(a_{2} / a_{1}\right)^{p}\right)$ it suffices to consider only matrices whose last column is identical to that of $C$. But comparing $B$ and $C$ it is clear that there is a bijection between these matrices, and so $a_{1} x \equiv x\left(a_{2} / a_{1}\right)^{p}$ modulo polynomials of degree less than $n+2-p$.

LEMMA 1.10. Suppose $x$ is a $p^{t}$ th power in $P[k]$. Then the admissible expression for $x$ in $R_{n}[k]^{*}$ is also a $p^{t}$ th power. Similarly, if $\psi^{-1}(I) \in \bigcap_{j=1}^{k-1} \operatorname{Ker} P^{\Delta_{k-1}}$, then $Q_{I}^{*} \in$ $\bigcap_{j=1}^{k-1} \operatorname{Ker} P^{\Delta_{k-1}}$.

Proof. To prove the first statement notice that, for each matrix $M \in \mathscr{S}(x)$ such that $M$ does not consist of groups of $p^{t}$ identical rows, $S(M)$ will occur a multiple of $p$ times by symmetry, so the result follows. For the second statement observe that, since application of $P^{\Delta_{j}}$ does not change polynomial degree for $j<k, x \in \operatorname{Ker} P^{\Delta_{j}}$ is equivalent to saying that the exponent of $a_{k-j}$ in each monomial of $x$ is divisible by $p$. Thus the proof of the second statement is the same, ignoring the rows of the matrices corresponding to the $a_{k}$-exponent on which no Adem relations are possible. 
Lemma 1.11. Suppose $x$ belongs to the principal ideal $\left(a_{k}^{r}\right)$ in $P[k]$. Then the admissible expression for $x$ in $R_{n}[k]^{*}$ is also in $\left(a_{k}^{r}\right)$.

Proof. There are no Adem relations possible on the last $r$ rows of the matrices and so, for each $M \in \mathscr{S}(x)$, the first entry of $S(M)$ is at least $r$.

2. Ann $P H^{*}\left(\Omega_{0}^{m+1} S^{m+1} ; Z / p Z\right)$. In this section we compute the elements of $P H^{*}\left(\Omega_{0}^{m+1} S^{m+1} ; Z / p Z\right)$ which are in the kernel of every Steenrod operation, denoted Ann $P H^{*}\left(\Omega_{0}^{m+1} S^{m+1} ; Z / p Z\right)$. We shall assume $m<\infty$ in this section since, if $m=\infty$, the $p$ th power operation is a monomorphism on $P[k]$ for each $k$, and thus Ann $P H^{*}\left(\Omega_{0}^{\infty} S^{\infty} ; Z / p Z\right)=0$.

Let

$$
x=\left(x_{k}\right) \in \operatorname{Ann} P H^{*}\left(\Omega_{0}^{m+1} S^{m+1} ; Z / p Z\right) \cong \bigoplus_{k} \operatorname{Ann}\left(R_{n}[k] / Q_{0} R_{n}[k]\right)^{*} .
$$

By Corollary $1.8, x_{k} \in j_{n}^{*}(P[k])$ for each $k$. We shall always assume that our elements are homogeneous. That is, $\left|x_{j}\right|=\left|x_{j^{\prime}}\right|$ for all $j$ and $j^{\prime}$, where || denotes homological degree.

Lemma 2.1. Let $x=\left(x_{k}\right) \in \oplus_{k} \operatorname{Ann}\left(R_{n}[k] / Q_{0} R_{n}[k]\right)^{*}$. Then $x$ has only one nonzero component.

Proof. Write $x=y^{p^{t}}$ where $y$ is not a $p$ th power. By Lemma 1.10 we may assume that this is the admissible expression for $x$. Since $x_{k} \in \bigcap_{j=1}^{k-1} \operatorname{Ker}\left(P^{\Delta_{j}}\right)^{p^{t}}$ and application of $P^{\Delta_{j}}$ does not affect polynomial degree, we must have $y_{k} \in$ $\cap_{j=1}^{k-1} \operatorname{Ker} P^{\Delta_{j}}$, so by Corollary 1.3 the exponent of $a_{j k}$ in each monomial of $y_{k}$ is divisible by $p$ for all $j<k$. Since $y$ is not a $p$ th power, for some $k$ the exponent of $a_{k k}$ in some monomial of $y_{k}$ is not divisible by $p$. But $\left|a_{1 k}^{r_{1}} \cdots a_{k k}^{r_{k}}\right| \equiv-2 r_{k}(\bmod p)$ $\left[r_{k}(\bmod 2)\right]$, and so since $x$ is homogeneous the exponent of $a_{k k}$ is not divisible by $p$ for each monomial of each $y_{k}$. Thus $\left(P^{\Delta_{k}}\right)^{p^{t}} x_{k}$ is nonzero back in $P[k]$, and the only way its image in $R_{n}[k]^{*}$ can be zero is if $\left(d\left(y_{k}\right)+1\right) p^{t}>n$, and so this inequality must hold for each $k$ such that $x_{k} \neq 0$.

Suppose $x_{j}, x_{k} \neq 0$ with $j<k$. Since $j<k,\left|a_{i j}\right|<\left|a_{r k}\right|$ for all $i$ and $r$. Thus since $\left|y_{j}\right|=\left|y_{k}\right|, d\left(y_{j}\right)>d\left(y_{k}\right)$. Thus $\left(d\left(y_{k}\right)+1\right) p^{t} \leqslant d\left(y_{j}\right) p^{t}=d\left(x_{j}\right) \leqslant n$, which contradicts the above inequality.

Let $W_{n}[k]=R_{n}[k] / Q_{0} R_{n}[k]$ modulo the subspace spanned by the sequences containing the Bockstein $\left[W_{n}[k]=R_{n}[k] / Q_{0} R_{n}[k]\right]$. Thus $W_{n}[k]^{*} \cong j_{n}^{*}\left(\left(a_{k}\right)\right)$ where $\left(a_{k}\right)=P[k] \cap I[k]$ is the principal ideal in $P[k]$ generated by $a_{k}$. Let $W_{n}=$ $\oplus_{k} W_{n}[k]$. By Corollary 1.8, $\operatorname{Ann}\left(R_{n}[k] / Q_{0} R_{n}[k]\right)^{*}=$ Ann $W_{n}[k]^{*}$. We compute Ann $W_{n}[k]^{*}$ by computing $W_{n}[k] / A_{*} W_{n}[k]$. Since $k$ and $n$ will be fixed for the remainder of this section, we will write simply $V$ for $W_{n}[k]$.

We find it convenient below to introduce an abbreviated notation which uses the symbol $x_{j}$. Unfortunately this conflicts with the standard notation used above in which $x_{k}$ denotes the $k$ th component of an element $x$ in a direct sum. Since, having fixed $k$ we have no further need in this section for the earlier notation, using the symbol $x_{j}$ differently for the remainder of this section should cause no confusion. 
If $x=Q_{2(p-1) I}\left[Q_{I}\right]$ for $I=\left(i_{k}, \ldots, i_{1}\right)$, let

$$
x_{j}=Q_{2(p-1)\left(i_{j}, \ldots, i_{1}\right)}\left[Q_{\left(i_{j}, \ldots, i_{1}\right)}\right], \quad 1 \leqslant j \leqslant k .
$$

Thus $x_{k}=x$. By convention, $\left|x_{0}\right|=0$. Notice that

$$
\begin{aligned}
\left|x_{j}\right| & =\left|x_{j-1}\right|+\frac{2 i_{j}+\left|x_{j-1}\right|}{2} 2(p-1) \\
& =2(p-1) i_{j}+p\left|x_{j-1}\right|=-2 i_{j}+p\left(2 i_{j}+\left|x_{j-1}\right|\right) \\
& =\left[\left|x_{j-1}\right|+i_{j}+\left|x_{j-1}\right|=-i_{j}+2\left(i_{j}+\left|x_{j-1}\right|\right)\right] .
\end{aligned}
$$

For $r \neq 0, r$ can be written uniquely as $r=u p^{t}$ with $u \neq 0(p)$. Let $\nu_{p}(r)=t$.

Suppose $1 \leqslant s \leqslant k$ and $t \geqslant 0$. We say that $Q_{2(p-1) I}\left[Q_{I}\right]$ satisfies $P(t, s)$ if

(1) $2 i_{s}+\left|x_{s-1}\right| \not \equiv 0\left(p^{t+1}\right)\left[i_{s}+\left|x_{s-1}\right| \not \equiv 0\left(2^{t+1}\right)\right]$, and

(2) $i_{s}+p^{t} \leqslant n$.

Intuitively, elements satisfying $P(t, s)$ for some $t$ and $s$ should be in $A_{*} V$, since the second condition shows that the element which we might expect to hit it under the Steenrod operation $P_{*}^{p^{t+k-s}}$ lies in $V$, and the first condition guarantees that the appropriate binomial coefficient in the Nishida relation is nonzero. The next theorem shows that this is in fact the case.

THEOREM 2.2. If $x=Q_{2(p-1) I}\left[Q_{I}\right]$ satisfies $P(t, s)$, then $x \in A_{*} V$. (Note: I need not be admissible.)

Proof. Order the pairs $(t, s)$ such that $1 \leqslant s \leqslant k, t \geqslant 0$, by $\left(t^{\prime}, s^{\prime}\right)<(t, s)$ if either $t^{\prime}-s^{\prime}<t-s$ or $t^{\prime}-s^{\prime}=t-s$ and $s^{\prime}<s$. The proof is by induction using this ordering.

To begin the induction suppose $x$ satisfies $P(0, k)$, which is the first $P(t, s)$ in the ordering. Let $J=I+(1,0, \ldots, 0)$ and let $y=Q_{2(p-1) J}\left[Q_{J}\right]$. Then $y \in V$ since $i_{k}+1 \leqslant n$ and

$$
P_{*}^{1} y=-\left(\begin{array}{c}
\left(2 i_{k}+\left|x_{k-1}\right|\right)((p-1) / 2) \\
1
\end{array}\right) x \quad\left[\left(\begin{array}{c}
i_{k}+\left|x_{k-1}\right| \\
1
\end{array}\right) x\right]
$$

Since $2 i_{k}+\left|x_{k-1}\right| \not \equiv 0(p)\left[i_{k}+\left|x_{k-1}\right| \not \equiv 0(2)\right]$, the coefficient is nonzero, so $x \in$ $A_{*} V$, as desired.

Suppose now that the theorem has been proved for all pairs $\left(t^{\prime}, s^{\prime}\right)$ such that $\left(t^{\prime}, s^{\prime}\right)<(t, s)$, and let $Q_{2(p-1) I}\left[Q_{I}\right]$ satisfy $P(t, s)$. We may suppose that $Q_{2(p-1) I}$ $\left[Q_{i}\right]$ fails to satisfy $P\left(t^{\prime}, s^{\prime}\right)$ for any $\left(t^{\prime}, s^{\prime}\right)$ such that $\left(t^{\prime}, s^{\prime}\right)<(t, s)$, because if it does we are finished by the induction hypothesis.

We consider first the case where $I$ is admissible.

Let $y=y_{k}=Q_{2(p-1) J}\left[Q_{J}\right]$ where $J=I+\left(0, \ldots, 0, p^{t}, 0, \ldots, 0\right)$ with the $p^{t}$ in the $s$ th position from the right. So $y_{j}=x_{j}$ for $1 \leqslant j<s$ and $\left|y_{j}\right|=\left|x_{j}\right|+2(p-1) p^{j-s+t}$ $\left[\left|y_{j}\right|=\left|x_{j}\right|+2^{j-s+t}\right]$ for $s \leqslant j \leqslant k$. 
LEMMA 2.3. $2 i_{s+j}+\left|x_{s+j-1}\right| \equiv 0\left(p^{t+j}\right)\left[i_{s+j}+\left|x_{s+j-1}\right| \equiv 0\left(2^{t+j}\right)\right], 0 \leqslant j \leqslant k$.

Proof. For $j=0$ we have $i_{s}+p^{t-1} \leqslant i_{s}+p^{t} \leqslant n$, so by failure to satisfy $P(t-1, s), 2 i_{s}+\left|x_{s-1}\right| \equiv O\left(p^{t}\right)\left[i_{s}+\left|x_{s-1}\right| \equiv 0\left(2^{t}\right)\right]$. Assume now that the lemma has been proved for $j^{\prime}<j$. Let $\nu_{p}\left(2 i_{s+1}+\left|x_{s+j-1}\right|\right)=r\left[\nu_{2}\left(i_{s+j}+\left|x_{s+j-1}\right|\right)=r\right]$, and suppose $r<t+j$. By the induction hypothesis, $2 i_{s+j-1}+\left|x_{s+j-2}\right| \equiv 0\left(p^{t+j-1}\right)$ $\left[i_{s+j-1}+\left|x_{s+j-2}\right| \equiv O\left(2^{t+j-1}\right)\right]$, and so, since

$$
\begin{gathered}
2 i_{s+j}+\left|x_{s+j-1}\right|=2\left(i_{s+j}-i_{s+j-1}\right)+p\left(2 i_{s+j-1}+\left|x_{s+j-2}\right|\right) \\
{\left[i_{s+j}+\left|x_{s+j-1}\right|=i_{s+j}-i_{s+j-1}+2\left(i_{s+j-1}+\left|x_{s+j-1}\right|\right)\right]}
\end{gathered}
$$

and $r+1 \leqslant t+j$, we get $\nu_{p}\left(i_{s+j}-i_{s+j-1}\right)=r$. Since $I$ is admissible, $i_{s+j} \leqslant i_{s+j-1}$ and so this implies $i_{s+j}+p^{r} \leqslant i_{s+j-1} \leqslant n$. But since $r<t+j$ and $Q_{2(p-1) I}\left[Q_{I}\right]$ fails to satisfy $P(r, s+j)$, we get $2 i_{s+j}+\left|x_{s+j-1}\right| \equiv 0\left(p^{r+1}\right)\left[i_{s+j}+\left|x_{s+j-1}\right| \equiv\right.$ $\left.0\left(2^{r+1}\right)\right]$, which is a contradiction. Therefore $r \geqslant t+j$, as desired.

\section{LEMMA 2.4.}

$$
\begin{gathered}
P_{*}^{p^{t+k-s}} y_{k} \equiv Q_{2(p-1)\left(j_{k}, \ldots j_{m+1}\right)} P_{*}^{p^{t+m-s}} y_{m} \quad \bmod A_{*} V \\
{\left[\mathrm{Sq}_{*}^{2^{t+k-s}} y_{k} \equiv Q_{\left(j_{k}, \ldots, j_{m+1}\right)} \mathrm{Sq}_{*}^{2^{t+m-s}} y_{m} \bmod A_{*} V\right]}
\end{gathered}
$$

for $s \leqslant m \leqslant k$. In particular,

$$
\begin{gathered}
P_{*}^{p^{t+k-s}} y_{k} \equiv Q_{2(p-1)\left(j_{k}, \ldots j_{s+1}\right)} P_{*} p^{t} y_{s} \quad \bmod A_{*} V \\
{\left[\mathrm{Sq}_{*}^{2^{t+k-s}} y_{k} \equiv Q_{\left(j_{k}, \ldots j_{s+1}\right)} \mathrm{Sq}_{*}^{2^{t}} y_{s} \quad \bmod A_{*} V\right]}
\end{gathered}
$$

Proof. The proof is by downward induction on $m$, the case $m=k$ being trivial. So we suppose the lemma holds for $m, m>s$, and show it for $m-1$. Let $r=t+m-s$.

$$
\begin{gathered}
P_{*}^{p^{r}} y_{m}=\sum_{l} c_{l} Q_{2(p-1)\left(j_{m}-p^{r}+p l\right)} P_{*}^{l} y_{m-1} \\
{\left[\mathrm{Sq}_{*}^{2^{r}} y_{m}=\sum_{l} c_{l} Q_{j_{m}-2^{r}+2 l} \mathrm{Sq}_{*}^{l} y_{m-1}\right]}
\end{gathered}
$$

where

$$
\begin{gathered}
c_{l}=(-1)^{p^{r+l}}\left(\begin{array}{c}
\left(2 j_{m}-2 p^{r}+\left|y_{m-1}\right|\right)\left(\frac{p-1}{2}\right) \\
p^{r}-p l
\end{array}\right) \\
{\left[c_{l}=\left(\begin{array}{c}
j_{m}+2^{r}+\left|y_{m-1}\right| \\
2^{r}-2 l
\end{array}\right)\right] .}
\end{gathered}
$$


Since

$$
\begin{aligned}
& 2 j_{m}-2 p^{r}+\left|y_{m-1}\right|=2 i_{m}+\left|y_{m-1}\right|-2 p^{r} \\
& =2 i_{m}+\left|x_{m-1}\right|+2(p-1) p^{m-1-2+t}-2 p^{r} \\
& =2 i_{m}+\left|x_{m-1}\right|+2(p-1) p^{r-1}-2 p^{r}=2 i_{m}+\left|x_{m-1}\right|-2 p^{r-1} \\
& {\left[j_{m}+\left|y_{m-1}\right|-2^{r}=i_{m}+\left|y_{m-1}\right|-2^{r}=i_{m}+\left|x_{m-1}\right|+2^{m-1-s+t}-2^{r}\right.} \\
& \left.=i_{m}+\left|x_{m-1}\right|+2^{r-1}-2^{r}=i_{m}+\left|x_{m-1}\right|-2^{r-1}\right],
\end{aligned}
$$

a direct application of Lemma 2.3 gives

$$
\begin{gathered}
\left(2 j_{m}-2 p^{r}+\left|y_{m-1}\right|\right)\left(\frac{p-1}{2}\right) \equiv-2 p^{r-1}\left(\frac{p-1}{2}\right) \equiv p^{r-1} \quad\left(\bmod p^{r}\right) \\
{\left[j_{m}-2^{r}+\left|y_{m-1}\right| \equiv 2^{r-1} \quad\left(\bmod 2^{r}\right)\right],}
\end{gathered}
$$

so a nonzero binomial coefficient is possible only when $l=0,(p-1) p^{r-2}$ or $p^{r-1}$. Since $c_{p^{r-1}}=1$, we get

$$
\begin{aligned}
Q_{2(p-1)\left(j_{k}, \ldots j_{m}\right)} P_{*}^{p^{r}} y_{m}= & c_{0} Q_{2(p-1)\left(j_{k}, \ldots j_{m+1}, j_{m}-p^{r}\right)} y_{m-1} \\
& +c_{(p-1) p^{r-2}} Q_{2(p-1)\left(j_{k}, \ldots j_{m+1}, j_{m}-(p-1) p^{r-1}\right)} P_{*}^{(p-1) p^{r-2} y_{m-1}} \\
& +Q_{2(p-1)\left(j_{k} \ldots j_{m}\right)} P_{*}^{p^{r-1}} y_{m-1}
\end{aligned}
$$

(3) $\left[Q_{\left(j_{k}, \ldots, j_{m}\right)} \mathrm{Sq}_{*}^{2^{r}} y_{m}=c_{0} Q_{\left(j_{k}, \ldots j_{m+1}, j_{m}-2^{r}\right)} y_{m-1}\right.$

$$
\left.+c_{2^{r-2}} Q_{\left(j_{k}, \ldots j_{m+1}, j_{m}-2^{r-1}\right)} \mathrm{Sq}_{*}^{2^{r-2}} y_{m-1}+Q_{\left(j_{k}, \ldots j_{m}\right)} \mathrm{Sq}_{*}^{2^{r-1}} y_{m-1}\right] \text {. }
$$

We will show that the first two terms are in $A_{*} V$.

For the first term consider

$$
\begin{gathered}
2 j_{s+1}+\left|y_{s}\right|=2 i_{s+1}+\left|x_{s}\right|+2(p-1) p^{t} \equiv-2 p^{t} \quad\left(\bmod p^{t+1}\right) \\
{\left[j_{s+1}+\left|y_{s}\right|=i_{s+1}+\left|x_{s}\right|+2^{t} \equiv 2^{t} \quad\left(\bmod 2^{t+1}\right)\right]}
\end{gathered}
$$

by Lemma 2.3. Also $j_{s+1}+p^{t}=i_{s+1}+p^{t} \leqslant i_{s}+p^{t} \leqslant n$. Thus for $m \geqslant s+2$, $Q_{2(p-1)\left(j_{k}, \ldots, j_{m+1}, j_{m}-p^{r}\right)} y_{m-1}\left[Q_{\left(j_{k}, \ldots, j_{m+1}, j_{m}-2^{r}\right)} y_{m-1}\right]$ satisfies $P(t, s+1)$, and thus is in $A_{*} V$. For $m=s+1$, the appropriate modification is

$$
\begin{gathered}
2 j_{s+1}-2 p^{r}+\left|y_{s}\right|=2 j_{s+1}-2 p^{t+1}+\left|y_{s}\right| \equiv-2 p^{t} \quad\left(\bmod p^{t+1}\right) \\
{\left[j_{s+1}-2^{r}+\left|y_{s}\right|=j_{s+1}-2^{t+1}+\left|y_{s}\right| \equiv 2^{t} \quad\left(\bmod 2^{t+1}\right)\right]}
\end{gathered}
$$

and $j_{s+1}-p^{r}+p^{t} \leqslant j_{s+1}+p^{t} \leqslant n$, so the same argument applies. Thus the first term is in $A_{*} V$.

For the second term consider the equation

$$
\begin{aligned}
& P_{*}^{(p-1) p^{r-2} y_{m-1}}=\sum_{l} b_{l} Q_{2(p-1)\left(j_{m-1}-(p-1) p^{r-2}+p^{l}\right)} P_{*}^{l} y_{m-2} \\
& {\left[\mathrm{Sq}_{*}^{2^{r-2}} y_{m-1}=\sum_{l} b_{l} Q_{j_{m-1}-2^{r-2}+2^{\prime}} \mathrm{Sq}_{*}^{l} y_{m-2}\right] \text {, }}
\end{aligned}
$$


where

$$
\begin{aligned}
b_{l}= & (-1)^{(p-1) p^{r-2}+l}\left(\begin{array}{c}
\left(2 j_{m-1}-2(p-1) p^{r-2}+\left|y_{m-2}\right|\right)\left(\frac{p-1}{2}\right) \\
(p-1) p^{r-2}-p l
\end{array}\right) \\
& \cdot\left[\left(\begin{array}{c}
j_{m-1}-2^{r-2}+\left|y_{m-2}\right| \\
2^{r-2}-2 l
\end{array}\right)\right]
\end{aligned}
$$

Look first at the case $m>s+1$. Then

$$
\begin{aligned}
\left(2 j_{m-1}-2(p-1) p^{r-2}+\left|y_{m-2}\right|\right)\left(\frac{p-1}{2}\right) & \\
= & \left(2 i_{m-1}-2(p-1) p^{r-2}+\left|x_{m-2}\right|+2(p-1) p^{r-2}\right)\left(\frac{p-1}{2}\right) \equiv 0\left(p^{r-1}\right) \\
& {\left[j_{m-1}-2^{r-2}+\left|y_{m-2}\right|=i_{m-1}-2^{r-2}+\left|x_{m-2}\right|+2^{r-2}\right] \equiv\left[0\left(2^{r-1}\right)\right] . }
\end{aligned}
$$

So for a nonzero binomial coefficient, $l=(p-1) p^{r-3}$. Continuing,

$$
\begin{gathered}
Q_{2(p-1)\left(j_{k}, \ldots, j_{m+1}, j_{m}-(p-1) p^{r-1}\right)} P_{*}^{(p-1) p^{r-2} y_{m-1}} \\
=\cdots=Q_{2(p-1)\left(j_{k}, \ldots j_{m+1}, j_{m}-(p-1) p^{r-1}, j_{m-1}\right)} P_{*}^{(p-1) p^{r-3}} y_{m-2} \\
=Q_{2(p-1)\left(j_{k}, \ldots j_{m+1}, j_{m}-(p-1) p^{r-1}, j_{m-1} \ldots, j_{s+2}\right)} P_{*}^{(p-1) p^{t} y_{s+1}} \\
{\left[Q_{\left(j_{k}, \ldots j_{m+1}, j_{m}-2^{r-1}\right)} \mathrm{Sq}_{*}^{2^{r-2} y_{m-1}}=Q_{\left(j_{k}, \ldots, j_{m+1}, j_{m}-2^{r-1}, j_{m-1}\right)} \mathrm{Sq}_{*}^{2^{r-3}} y_{m-2}\right.} \\
\left.=\cdots=Q_{\left(j_{k}, \ldots j_{m+1}, j_{m}-2^{r-1}, j_{m-1}, \ldots j_{s+2}\right)} \mathrm{Sq}_{*}^{2^{t} y_{s+1}}\right] .
\end{gathered}
$$

Now if $m \geqslant s+3$,

$$
\begin{aligned}
& 2 j_{s+2}+\mid P_{*}^{(p-1) p^{t} y_{s+1} \mid}=2 i_{s+2}+\left|y_{s+1}\right|-2(p-1)^{2} p^{t} \\
&=2 i_{s+2}+\left|x_{s+1}\right|+2(p-1) p^{t+1}-2(p-1)^{2} p^{t} \\
&=2 i_{s+2}+\left|x_{s+1}\right|+2(p-1) p^{t} \equiv-2 p^{t} \quad\left(\bmod p^{t+1}\right) \\
& {\left[j_{s+2}+\mid \mathrm{Sq}_{*}^{2^{t} y_{s+1}\left|=i_{s+2}+\right| y_{s+1} \mid-2^{t}}\right.} \\
&\left.=i_{s+2}+\left|x_{s+1}\right|+2^{t+1}-2^{t} \equiv 2^{t} \quad\left(\bmod 2^{t+1}\right)\right]
\end{aligned}
$$

and $j_{s+2} \leqslant i_{s+2}+p^{t} \leqslant i_{s}+p^{t} \leqslant n$, so this term satisfies $P(t, s+2)$ and thus is in $A_{*} V$. If $m=s+2$,

$$
j_{s+2}-(p-1) p^{r-1}=j_{s+2}-(p-1) p^{t+1} \equiv j_{s+2} \quad\left(\bmod p^{t+1}\right)
$$

and $j_{s+2}-(p-1) p^{r-1} \leqslant j_{s+2}$, so the argument is the same. Finally, if $m=s+1$, returning to equation (3),

$$
Q_{2(p-1)\left(j_{k}, \ldots, j_{s+2}, j_{s+1}-(p-1) p^{t}\right)} P_{*}^{(p-1) p^{t-1}} y_{s} \quad\left[Q_{\left(j_{k}, \ldots, j_{s+2}, j_{s+1}-2^{t}\right)} \mathrm{Sq}_{*}^{2^{2-1}} y_{s}\right]
$$


satisfies

$$
\begin{aligned}
& 2 j_{s+1}-2(p-1) p^{t}+\left|P_{*}^{(p-1) p^{t-1}} y_{s}\right|=2 i_{s+1}-2(p-1) p^{t}+\left|y_{s}\right|+2(p-1)^{2} p^{t-1} \\
& =2 i_{s+1}-2(p-1) p^{t}+\left|x_{s}\right|+2(p-1) p^{t}-2(p-1)^{2} p^{t-1} \\
& \equiv-2(p-1)^{2} p^{t-1} \not \equiv 0\left(p^{t+1}\right) \\
& {\left[i_{s+1}-2^{t}+\left|\mathrm{Sq}_{*}^{2^{t-1}} y_{s}\right|=i_{s+1}-2^{t}+\left|y_{s}\right|-2^{t-1}\right.} \\
& \left.=i_{s+1}-2^{t}+\left|x_{s}\right|+2^{t}-2^{t-1} \equiv-2^{t-1} \not \equiv 0\left(2^{t+1}\right)\right]
\end{aligned}
$$

and $j_{s+1}-(p-1) p^{t}+p^{t} \leqslant j_{s+1}=i_{s+1} \leqslant n$, so the term satisfies $P(t, s+1)$ and thus is in $A_{*} V$.

So in all cases the second term also lies in $A_{*} V$ and the lemma is proved.

Proof of Theorem 2.2 (CONTINUED).

$$
\begin{gathered}
P_{*}^{p^{t}} y_{s}=\sum_{l} c_{l} Q_{2(p-1)\left(j_{s}-p^{t}+p l\right)} P_{*}^{l} y_{s-1}=\sum_{l} c_{l} Q_{2(p-1)\left(i_{s}+p l\right)} P_{*}^{l} y_{s-1} \\
{\left[\mathrm{Sq}_{*}^{2^{l}} y_{s}=\sum_{l} c_{l} Q_{j_{s}-2^{t}+2 l} \mathrm{Sq}_{*}^{l} y_{s-1}=\sum_{l} c_{l} Q_{i_{s}+2 l} \mathrm{Sq}_{*}^{l} x_{s-1}\right]}
\end{gathered}
$$

where

$$
c_{l}=(-1)^{p^{t}+l}\left(\begin{array}{c}
\left(2 j_{s}-2 p^{t}+\left|y_{s-1}\right|\right)\left(\frac{p-1}{2}\right) \\
p^{t}-p l
\end{array}\right)\left[\left(\begin{array}{c}
j_{s}-2^{t}+\left|y_{s-1}\right| \\
2^{t}-2 l
\end{array}\right)\right] .
$$

Since

$$
\begin{aligned}
& \nu_{p}\left(2 j_{s}-2 p^{t}+\left|y_{s-1}\right|\right)=\nu_{p}\left(2 i_{s}+\left|x_{s-1}\right|\right)=t \\
& {\left[\nu_{2}\left(j_{s}-2^{t}+\left|y_{s-1}\right|\right)=\nu_{2}\left(i_{s}+\left|x_{s-1}\right|\right)=t\right],}
\end{aligned}
$$

$c_{0} \neq 0, c_{p^{t-1}}=1$ and the other coefficients are zero. Thus

$$
\begin{gathered}
P_{*}^{p^{t+k-s} y_{k}} \equiv Q_{2(p-1)\left(j_{k}, \ldots j_{s+1}\right)} P_{*}^{p^{t}} y_{s} \\
=c_{0} x+Q_{2(p-1)\left(j_{k}, \ldots j_{s}\right)} P_{*}^{p^{t-1}} x_{s-1} \bmod A_{*} V \\
{\left[\mathrm{Sq}_{*}^{2^{t+k-s}} y_{k} \equiv Q_{\left(j_{k}, \ldots, j_{s+1}\right)} \mathrm{Sq}_{*}^{2^{t}} y_{s}=x+Q_{\left(j_{k}, \ldots, j_{s}\right)} \mathrm{Sq}_{*}^{2^{t-1}} x_{x-1} \bmod A_{*} V\right] .}
\end{gathered}
$$

It remains to show that the second term is zero modulo $A_{*} V$. Now

$$
\begin{aligned}
P_{*}^{p^{t-1}} x_{s-1} & =\sum_{m_{1}} b_{m_{1}} Q_{2(p-1)\left(i_{s-1}-p^{t-1}+p m_{1}\right)} P_{*}^{m_{1}} x_{s-2} \\
& =\sum_{m_{1}} \sum_{m_{2}} b_{m_{1}} b_{m_{2}} Q_{2(p-1)\left(i_{s-1}-p^{t-1}+p m_{1}\right)} Q_{2(p-1)\left(i_{s-2}-m_{1}+p m_{2}\right)} P_{*}^{m_{2}} x_{s-3} \\
{\left[\mathrm{Sq}_{*}^{2^{t-1}} x_{s-1}=\right.} & \sum_{m_{1}} b_{m_{1}} Q_{i_{s-1}-2^{t-1}+2 m_{1}} \mathrm{Sq}_{*}^{m_{1}} x_{s-2} \\
= & \left.\sum_{m_{1}} \sum_{m_{2}} b_{m_{1}} b_{m_{2}} Q_{i_{s-1}-2^{t-1}+2 m_{1}} Q_{i_{s-2}-m_{1}+2 m_{2}} \mathrm{Sq}_{*}^{m_{2}} x_{s-3}\right] .
\end{aligned}
$$


Continuing the expansion we get a summation of terms of the form

$$
\begin{gathered}
b_{m_{1}} \cdots b_{m_{\alpha+1}} Q_{2(p-1)\left(i_{s-1}-p^{t-1}+p m_{1}, i_{s-2}-m_{1}+p m_{2}, \ldots, i_{s-\alpha+1}-m_{\alpha-1}+p m_{\alpha}, i_{s-\alpha}-m_{\alpha}, i_{s-\alpha-1} \ldots, i_{1}\right)} \\
{\left[b_{m_{1}} \cdots b_{m_{\alpha+1}} Q_{\left(i_{s-1}-2^{t-1}+2 m_{1}, i_{s-2}-m_{1}+2 m_{3} \ldots, i_{s-\alpha+1}-m_{\alpha-1}+2 m_{\alpha}, i_{s-\alpha}-m_{\alpha}, i_{s-\alpha-1}, \ldots, i_{1}\right)}\right]}
\end{gathered}
$$

with $m_{0}, m_{1}, \ldots, m_{\alpha} \neq 0, m_{\alpha+1}=0,0 \leqslant \alpha \leqslant s$ (where $m_{0}=p^{t-1}$ by convention). $m_{\beta+1} \leqslant p m_{\beta}$ and so $m_{\beta} \leqslant p^{t-\beta-1}$ for $0 \leqslant \beta \leqslant \alpha$. In particular, $m_{\alpha} \leqslant p^{t-\alpha-1}$. Find $\gamma$ such that $p^{\gamma} \leqslant m_{\alpha}<p^{\gamma+1}$. So $0 \leqslant \gamma \leqslant t-\alpha-1$. Since $i_{s-\alpha}-m_{\alpha}+p^{\gamma} \leqslant i_{s-\alpha} \leqslant n$, if $2 i_{s-\alpha}-2 m_{\alpha}+\left|x_{s-\alpha-1}\right| \equiv 0\left(p^{\gamma+1}\right)\left[i_{s-\alpha}-m_{\alpha}+\left|x_{s-\alpha-1}\right| \neq \equiv 0\left(2^{\gamma+1}\right)\right]$, then $P(\gamma, s-\alpha-1)$ would be satisfied and so, since $\gamma \leqslant t-\alpha-1$, the induction hypothesis would imply that the term is in $A_{*} V$. On the other hand, if $2 i_{s-\alpha}-2 m_{\alpha}$ $+\left|x_{s-\alpha-1}\right| \equiv 0\left(p^{\gamma+1}\right)\left[i_{s-\alpha}-m_{\alpha}+\left|x_{s-\alpha-1}\right| \equiv 0\left(2^{\gamma+1}\right)\right]$, then the binomial coefficient

$$
b_{\alpha+1}= \pm\left(\begin{array}{c}
\left(2 i_{s-\alpha}-2 m_{\alpha}+\left|x_{s-\alpha-1}\right|\right)((p-1) / 2) \\
m_{\alpha}
\end{array}\right)\left[\left(\begin{array}{c}
\left.i_{s-\alpha}-m_{\alpha}+\left|x_{s-\alpha-1}\right|\right) \\
m_{\alpha}
\end{array}\right)\right]
$$

is zero since $0<m_{\alpha}<p^{\gamma+1}$.

We have just shown that any admissible monomial satisfying $P(t, s)$ is in $A_{*} V$. Now suppose that $x=Q_{2(p-1) I}\left[Q_{I}\right]$ satisfies $P(t, s)$ but is not necessarily admissible. Use the Adem relations to write $Q_{2(p-1) I}\left[Q_{I}\right]$ admissibly. We conclude the proof of the theorem by showing that, modulo terms already known to be in $A_{*} V$ from the induction hypothesis, all the resulting terms in the admissible expression for $Q_{2(p-1) I}\left[Q_{I}\right]$ satisfy $P(t, s)$.

We may assume that $Q_{2(p-1) I}\left[Q_{I}\right]$ fails to satisfy $P(t-1, s)$, and so as in the proof of Lemma 2.3 we conclude that $\nu_{p}\left(2 i_{s}+\left|x_{s-1}\right|\right)=t\left[\nu_{2}\left(i_{s}+\left|x_{s-1}\right|\right)=t\right]$.

Using the Adem relations to rewrite any of the factors other than $Q_{2(p-1) i_{s+1}} Q_{2(p-1) i_{s}}\left[Q_{i_{s+1}} Q_{i_{s}}\right]$ or $Q_{2(p-1) i_{s}} Q_{2(p-1) i_{s-1}}\left[Q_{i_{s}} Q_{i_{s-1}}\right]$ does not affect the condition $P(t, s)$, and so the resulting terms must therefore still satisfy this condition. Thus it remains to show that the condition $P(t, s)$ is maintained after applying Adem relations in these two positions.

Suppose $Q_{2(p-1) i_{s}} Q_{2(p-1) i_{s-1}}\left[Q_{i_{s}} Q_{i_{s-1}}\right]$ is inadmissible; that is, $i_{s}>i_{s-1}$. Thus $i_{s-1}+p^{t-1} \leqslant i_{s}+p^{t-1} \leqslant n$, so we must have $2 i_{s-1}+\left|x_{s-2}\right| \equiv 0\left(p^{t}\right)\left[i_{s-1}+\left|x_{s-2}\right|\right.$ $\left.\equiv 0\left(2^{t}\right)\right]$ or else $P(t-1, s-1)$ would be satisfied, which would imply $Q_{2(p-1) I}$ $\left[Q_{I}\right] \in A_{*} V$.

$$
\begin{gathered}
Q_{2(p-1) i_{s}} Q_{2(p-1) i_{s-1}}=\sum_{j} c_{j} Q_{2(p-1)\left(i_{s}+p i_{s-1}-p j\right)} Q_{2(p-1) j} \\
{\left[Q_{i_{s}} Q_{i_{s-1}}=\sum_{j} c_{j} Q_{i_{s}+2 i_{s-1}-2 j} Q_{j}\right]}
\end{gathered}
$$

where

$$
c_{j}=(-1)^{i_{s}+(p-1) i_{s-1}+j}\left(\begin{array}{c}
\left(j-i_{s-1}\right)(p-1)-1 \\
i_{s}-j-1
\end{array}\right) .
$$


We wish to show that

$$
\begin{gathered}
c_{j} Q_{2(p-1)\left(i_{k}, \ldots, i_{s+1}, i_{s}+p i_{s-1}-p j, j\right)} x_{s-2} \\
{\left[c_{j} Q_{\left(i_{k}, \ldots, i_{s+1}, i_{s}+2 i_{s-1}-2 j, j\right)} x_{s-2}\right]}
\end{gathered}
$$

satisfies $P(t, s)$ modulo terms in $A_{*} V$. Now

$$
\begin{aligned}
2 i_{s}+2 p i_{s-1}-2 p j+\left|Q_{2(p-1) j} x_{s-2}\right| & \\
= & 2 i_{s}+2 p i_{s-1}-2 p j+2(p-1) j+p\left|x_{s-2}\right| \\
= & 2 i_{s}-2 j+p\left(2 i_{s-1}+\left|x_{s-2}\right|\right) \equiv 2 i_{s}-2 j\left(p^{t+1}\right) \\
{\left[i_{s}+2 i_{s-1}-2 j+\left|Q_{j} x_{s-2}\right|\right.} & =i_{s}+2 i_{s-1}-2 j+j+2\left|x_{s-2}\right| \\
& \left.=i_{s}-j+2\left(i_{s-1}+\left|x_{s-2}\right|\right) \equiv i_{s}-j\left(2^{t+1}\right)\right] .
\end{aligned}
$$

Suppose $i_{s}-j \equiv 0\left(p^{t+1}\right)$. Then $i_{s}-j-1 \equiv-1\left(p^{t+1}\right)$, so for a nonzero binomial coefficient, $\left(j-i_{s-1}\right)(p-1)-1 \equiv-1\left(p^{t+1}\right)$ and so $j-i_{s-1} \equiv O\left(p^{t+1}\right)$. But then

$$
\begin{array}{rl}
2 i_{s}+\left|x_{s-1}\right| & =2 i_{s}-2 i_{s-1}+p\left(2 i_{s-1}+\left|x_{s-2}\right|\right) \\
=2 & 2\left(i_{s}-j\right)+2\left(j-i_{s-1}\right)+p\left(2 i_{s-1}+\left|x_{s-2}\right|\right) \equiv 0\left(p^{t+1}\right) \\
{\left[i_{s}+\left|x_{s-1}\right|=i_{s}-i_{s-1}+\right.} & 2\left(i_{s-1}+\left|x_{s-2}\right|\right) \\
= & \left.\left(i_{s}-j\right)+\left(j-i_{s-1}\right)+2\left(i_{s-1}+\left|x_{s-2}\right|\right) \equiv 0\left(2^{t+1}\right)\right],
\end{array}
$$

contradicting the hypothesis on $Q_{2(p-1) I}\left[Q_{I}\right]$. Thus $i_{s}-j \not \equiv 0\left(p^{t+1}\right)$, and so the first condition of $P(t, s)$ is satisfied. Since $j \geqslant i_{s-1}-1$ for a nonzero binomial coefficient, $i_{s}+p i_{s-1}-p j+p^{t}<i_{s}+p^{t} \leqslant n$, and so the other condition of $P(t, s)$ is also satisfied. Thus $P(t, s)$ is still satisfied after rewriting $Q_{2(p-1) i_{s}} Q_{2(p-1) i_{s-1}}$ $\left[Q_{i_{s}} Q_{i_{s-1}}\right]$.

Finally, suppose $Q_{2(p-1) i_{s+1}} Q_{2(p-1) i_{s}}\left[Q_{i_{s+1}} Q_{i_{s}}\right]$ is inadmissible.

$$
\begin{gathered}
Q_{2(p-1) i_{s+1}} Q_{2(p-1) i_{s}}=\sum_{j} c_{j} Q_{2(p-1)\left(i_{s+1}+p i_{s}-p j\right)} Q_{2(p-1) j} \\
{\left[Q_{i_{s+1}} Q_{i_{s}}=\sum_{j} c_{j} Q_{i_{s+1}+2 i_{s}-2 j} Q_{j}\right]}
\end{gathered}
$$

where

$$
c_{j}=(-1)^{i_{s+1}+(p-1) i_{s-1}+j}\left(\begin{array}{c}
\left(j-i_{s}\right)(p-1)-1 \\
i_{s+1}-j-1
\end{array}\right) \text {. }
$$

To show

$$
Q_{2(p-1)\left(i_{k}, \ldots, i_{s+2}, i_{s+1}+p i_{s}-p j, j\right)} x_{s-1} \quad\left[Q_{\left(i_{k}, \ldots, i_{s+2}, i_{s+1}+2 i_{s}-2 j, j\right)} x_{j-1}\right]
$$

satisfies $P(t, s)$ we need $2 j+\left|x_{s-1}\right| \not \equiv O\left(p^{t+1}\right)\left[j+\left|x_{s-1}\right| \not \equiv O\left(2^{t+1}\right)\right]$ and $j+p^{t} \leqslant$ $n$. 
Suppose $i_{s+1}-j \neq \equiv\left(p^{t+1}\right)$. Let $\nu_{p}\left(i_{s+1}-j\right)=r$. Thus $r \leqslant t$. For a nonzero binomial coefficient we must have $j-i_{s} \equiv 0\left(p^{r}\right)$. Now

$$
\begin{aligned}
2 i_{s+1}+2 p i_{s}-2 p j+ & \left|Q_{2(p-1) j} x_{s-1}\right| \\
& =2 i_{s+1}+2 p i_{s}-2 p j+2(p-1) j+p\left|x_{s-1}\right| \\
=2 i_{s+1}-2 j+p\left(2 i_{s}+\left|x_{s-1}\right|\right) \equiv 2\left(i_{s-1}-j\right) \not \equiv 0\left(p^{r+1}\right) & \\
{\left[i_{s+1}+2 i_{s}-2 j+\left|Q_{j} x_{s-1}\right|\right.} & =i_{s+1}+2 i_{s}-2 j+j+2\left|x_{s-1}\right| \\
& \left.=i_{s+1}-j+2\left(i_{s}+\left|x_{s-1}\right|\right) \equiv i_{s+1}-j \neq 0\left(2^{r+1}\right)\right]
\end{aligned}
$$

since $r \leqslant t$. So the first condition of $P(r, s+1)$ is satisfied. From the fact that $Q_{2(p-1)\left(i_{s+1}+p i_{s}-p_{j}\right)} Q_{2(p-1) j}\left[Q_{i_{s+1}+2 i_{s}-2 j} Q_{j}\right]$ is admissible, $j-\left(i_{s+1}+p i_{s}-p j\right) \geqslant 0$. However,

$$
\nu_{p}\left(j-\left(i_{s+1}-p i_{s}-p j\right)\right)=\nu_{p}\left(j-i_{s+1}+p\left(i_{s}-j\right)\right)=\nu_{p}\left(j-i_{s+1}\right)=r,
$$

and so $j-\left(i_{s+1}-p i_{s}-p j\right) \geqslant p^{r}$. Thus $i_{s+1}+p i_{s}-p j+p^{r} \leqslant j<i_{s+1} \leqslant n$ and so $P(r, s+1)$ is satisfied. Since $r \leqslant t$ this implies that the term is in $A_{*} V$ by the induction hypothesis.

Thus all terms with $i_{s+1}-j \neq \equiv\left(p^{t+1}\right)$ are in $A_{*} V$. But if $i_{s+1}-j \equiv O\left(p^{t+1}\right)$, then for a nonzero binomial coefficient, $j-i_{s} \equiv 0\left(p^{t+1}\right)$, and so

$$
\begin{aligned}
& 2 j+\left|x_{s-1}\right|=2\left(j-i_{s}\right)+\left(2 i_{s}+\left|x_{s-1}\right|\right) \equiv 2 i_{s}+\left|x_{s-1}\right| \not \equiv 0\left(p^{t+1}\right) \\
& {\left[j+\left|x_{s-1}\right|=\left(j-i_{s}\right)+\left(i_{s}+\left|x_{s-1}\right|\right) \equiv i_{s}+\left|x_{s-1}\right| \not \equiv 0\left(2^{t+1}\right)\right]}
\end{aligned}
$$

so the first condition of $P(t, s)$ is satisfied. Also $j \leqslant i_{s+1}-1$, and so $i_{s+1}-j \equiv$ $0\left(p^{t+1}\right)$ implies $j+p^{t+1} \leqslant i_{s+1}$. Thus $j+p^{t} \leqslant i_{s+1} \leqslant n$, and so the second condition of $P(t, s)$ is satisfied.

Thus any inadmissible monomial satisfying $P(t, s)$ can be rewritten as a sum of admissible monomials satisfying $P(t, s)$ modulo terms in $A_{*} V$ and so the proof of the theorem is complete.

Let $N_{n}[k]=\left\{Q_{2(p-1) I}\left[Q_{I}\right] \in W_{n}[k] \mid Q_{2(p-1) I}\left[Q_{I}\right]\right.$ is admissible and fails to satisfy $P(t, s)$ for any $t$ and $s, t \geqslant 0,1 \leqslant s \leqslant k\}$. That is. $N_{n}[k]=\left\{Q_{2(p-1) I}\right.$ $\left[Q_{I}\right] \in W_{n}[k] \mid Q_{2(p-1) I}\left[Q_{I}\right]$ is admissible and $i_{s}+p^{t} \leqslant n$ implies $2 i_{s}+\left|x_{s-1}\right| \equiv$ $0\left(p^{t+1}\right)\left[i_{s}+\left|x_{s-1}\right| \equiv 0\left(2^{t+1}\right)\right]$ for all $t \geqslant 0$ and $\left.1 \leqslant s \leqslant k\right\}$.

The remainder of this section will be devoted to a more detailed description of $N_{n}[k]$ and to the proof of the following theorem.

THEOREM 2.5. $\left\{Q_{2(p-1) I}^{*} \mid Q_{2(p-1) I} \in N_{n}[k]\right\}\left[\left\{Q_{I}^{*} \mid Q_{I} \in N_{n}[k]\right\}\right]$ forms a basis for $\operatorname{Ann}\left(R_{n}[k] / Q_{0} R_{n}[k]\right)^{*}$.

We first show that if $Q_{2(p-1) I}\left[Q_{I}\right] \in N_{n}[k]$ then $I$ is completely determined by $i_{1}$ and $i_{k}$, and thus there is at most one $Q_{2(p-1) I}\left[Q_{I}\right] \in N_{n}[k]$ with a given $i_{1}$ and $i_{k}$. 
LeMma 2.6. Let $Q_{2(p-1) I}\left[Q_{I}\right] \in N_{n}[k]$. Suppose $\nu_{p}\left(2 i_{s}+\left|x_{s-1}\right|\right)=t\left[\nu_{2}\left(i_{s}+\left.\right|_{s-1} \mid\right)\right.$ $=t$ ] for some $s, 1 \leqslant s \leqslant k-1$. Then $i_{s}-i_{s+1} \equiv 0\left(p^{t+1}\right)$ and $2 i_{s+1}+\left|x_{s}\right| \equiv 0\left(p^{t+1}\right)$ $\left[i_{s+1}+\left|x_{s}\right| \equiv 0\left(2^{t+1}\right)\right]$. In particular, $s<s^{\prime}$ implies

$$
\begin{gathered}
\nu_{p}\left(2 i_{s}+\left|x_{s-1}\right|\right)<\nu_{p}\left(2 i_{s^{\prime}}+\left|x_{s^{\prime}-1}\right|\right) \\
{\left[\nu_{2}\left(i_{s}+\left|x_{s-1}\right|\right)<\nu_{2}\left(i_{s^{\prime}}+\left|x_{s}\right|\right)\right] .}
\end{gathered}
$$

Proof. Either $i_{s}-i_{s+1}=0$ or $\nu_{p}\left(i_{s}-i_{s+1}\right)=r$ for some $r$. If the latter, then $i_{s+1}+p^{r} \leqslant i_{s} \leqslant n$ and because $Q_{2(p-1) I}\left[Q_{I}\right] \in N_{n}[k], 2 i_{s+1}+\left|x_{s}\right| \equiv 0\left(p^{r+1}\right)\left[i_{s}+\right.$ $\left.\left|x_{s}\right| \equiv 0\left(2^{r+1}\right)\right]$. However, $2 i_{s+1}+\left|x_{s}\right|=2\left(i_{s+1}-i_{s}\right)+p\left(2 i_{s}+\left|x_{s-1}\right|\right)\left[i_{s+1}+\left|x_{s}\right|\right.$ $\left.=i_{s+1}-i_{s}+2\left(i_{s}+\left|x_{s-1}\right|\right)\right]$ and so $r \geqslant t+1$. Thus in either case, $i_{s}-i_{s+1} \equiv$ $0\left(p^{t+1}\right)$ and the above equation therefore implies that $2 i_{s+1}+\left|x_{s}\right| \equiv 0\left(p^{t+1}\right)\left[i_{s+1}\right.$ $\left.+\left|x_{s}\right| \equiv 0\left(2^{t+1}\right)\right]$.

LEMMA 2.7. Let $Q_{2(p-1) I}\left[Q_{I}\right] \in N_{n}[k]$. If up occurs in the p-adic expansion of $i_{s-1}-i_{s}$ for some $s, 2 \leqslant s \leqslant k, 1 \leqslant u<p$, then $2 i_{s}+\left|x_{s-1}\right| \equiv 0\left(p^{t+1}\right)\left[i_{s}+\left|x_{s-1}\right|\right.$ $\left.\equiv 0\left(2^{t+1}\right)\right]$ and $2 i_{s-1}+\left|x_{s-2}\right| \not \equiv 0\left(p^{t+1}\right)\left[i_{s-1}+\left|x_{s-2}\right| \not \equiv 0\left(2^{t+1}\right)\right]$.

Proof. Since $u p^{t}$ occurs in the $p$-adic expansion of $i_{s-1}-i_{s}, i_{s}+p^{t} \leqslant i_{s-1} \leqslant n$, so $2 i_{s}+\left|x_{s-1}\right| \equiv 0\left(p^{t+1}\right)\left[i_{s}+\left|x_{s-d 1}\right| \equiv 0\left(2^{t+1}\right)\right]$ by definition of $N_{n}[k]$. Now $2 i_{s}+\left|x_{s-1}\right|=2\left(i_{s}-i_{s-1}\right)+p\left(2 i_{s-1}+\left|x_{s-2}\right|\right)\left[i_{s}+\left|x_{s-1}\right|=i_{s}-i_{s-1}+\right.$ $\left.2\left(i_{s-1}+\left|x_{s-2}\right|\right)\right]$, so $p\left(2 i_{s-1}+\left|x_{s-2}\right|\right) \equiv 2\left(i_{s-1}-i_{s}\right) \neq 0\left(p^{t+1}\right)\left[2\left(i_{s-1}+\left|x_{s-2}\right|\right) \equiv\right.$ $\left.i_{s-1}-i_{s} \not \equiv 0\left(2^{t+1}\right)\right]$. Thus $2 i_{s-1}+\left|x_{s-2}\right| \not \equiv 0\left(p^{t+1}\right)\left[i_{s-1}+\left|x_{s-2}\right| \not \equiv 0\left(2^{t+1}\right)\right]$.

COROllary 2.8. Let $Q_{2(p-1) I}\left[Q_{I}\right] \in N_{n}[k]$.

(a) $u p^{t}$ occurs in the $p$-adic expansion of $i_{s-1}-i_{s}$ for at most one $s, 2 \leqslant s \leqslant k$, $1 \leqslant u<p$.

(b) Suppose up $p^{t}$ and $u^{\prime} p^{t^{\prime}}$ occur in the p-adic expansions of $i_{s-1}-i_{s}$ and $i_{s^{\prime}-1}-i_{s^{\prime}}$, respectively, $1 \leqslant u, u^{\prime}<p$. If $t<t^{\prime}$ then $s \leqslant s^{\prime}$.

(c) $u p^{t}$ occurs in the $p$-adic expansion of $i_{s-1}-i_{s}$ for some $s, 2 \leqslant s \leqslant k$, if and only if up ${ }^{t}$ occurs in the $p$-adic expansion of $i_{1}-i_{k}$.

Proof. (a) The $s$ for which the statement holds is characterized by Lemma 2.7 and this condition is satisfied by at most one $x$ by Lemma 2.6 .

(b) This is immediate from Lemmas 2.6 and 2.7 .

(c) Since there are no repetitions by (a), the set of $u p^{t}$ occurring in the $p$-adic expansion of $i_{s-1}-i_{s}$ for some $s, 2 \leqslant s \leqslant k$, forms the $p$-adic expansion of their $\operatorname{sum} \sum_{s=2}^{k} i_{s-1}-i_{s}=i_{1}-i_{k}$.

Let $i_{1}-i_{k}=u_{1} p^{t_{1}}+u_{2} p^{t_{2}}+\cdots+u_{q} p^{t_{q}}$ be the $p$-adic expansion of $i_{1}-i_{k}$, where $t_{1}<t_{2}<\cdots<t_{q}$ and $1 \leqslant u_{i}<p$. Let $\phi(m)$ den0te the unique $s$ for which $i_{s-1}-i_{s}$ contains $u_{m} p^{t_{m}}$ in its $p$-adic expansion. So $\phi$ is monotonically increasing by (b) of Corollary 2.8. We wish to show that $\phi$ (and thus $I$ ) is completely determined by $i_{1}$ and $i_{k}$. Suppose that $\phi(1), \ldots, \phi(m-1)$ have been computed in terms of $i_{1}$ and $i_{k}$. Set $s=\phi(m-1)$ and find $j$ such that $\phi(j-1)<s, \phi(j)=\cdots=\phi(m-1)=s$. (If $m=1$, set $s=2$.) So $i_{1}, \ldots, i_{s-1}$ (and thus $x_{1}, \ldots, x_{s-1}$ ) have already been computed in terms of $i_{1}$ and $i_{k}$. We have not yet determined $s$, but it must satisfy 


$$
\begin{gathered}
i_{s-1}-i_{s} \equiv u_{j} p^{t_{j}}+\cdots+u_{m-1} p^{t_{m-1}}\left(\bmod p^{t_{m}}\right) . \text { Write } \\
\begin{array}{c}
p\left(2 i_{s-1}+\left|x_{s-2}\right|\right) / 2+u_{j} p^{t_{j}}+\cdots+u_{m-1} p^{t_{m-1}}=v p^{r} \\
{\left[2\left(i_{s-1}+\left|x_{s-2}\right|\right)+2^{t_{j}}+\cdots+2^{t_{m-1}}=v 2^{r}\right]}
\end{array}
\end{gathered}
$$

with $v \not \equiv 0(p)$. Since the left-hand side has been computed in terms of $i_{1}$ and $i_{k}, r$ is determined by $i_{1}$ and $i_{k}$.

LEMMA 2.9. $\phi(m)=s+t_{m}-r$ provided $t_{m} \geqslant r$. If $t_{m}<r$ or if $s+t_{m}-r>k$, there does not exist $Q_{2(p-1) J}\left[Q_{J}\right] \in N_{n}[k]$ with this $i_{1}$ and $i_{k}$.

Proof. We know $\phi(m) \geqslant s$ since $\phi$ is monotonically increasing. If $\phi(m)=s$, then $i_{s-1}-i_{s} \equiv u_{j} p^{t_{j}}+\cdots+u_{m-1} p^{t_{m-1}}+u_{m} p^{t_{m}}\left(\bmod p^{t_{m}+1}\right)$, and so

$$
\begin{gathered}
2 i_{s}+\left|x_{s-1}\right|=2\left(i_{s}-i_{s-1}\right)+p\left(2 i_{s-1}+\left|x_{s-2}\right|\right) \equiv 2 u_{m} p^{t_{m}}+2 v p^{r} \quad\left(\bmod p^{t_{m}+1}\right) \\
{\left[i_{s}+\left|x_{s-1}\right|=i_{s}-i_{s-1}+2\left(i_{s-1}+\left|x_{s-2}\right|\right) \equiv 2^{t_{m}}+v 2^{r} \quad\left(\bmod 2^{t_{m}+1}\right)\right] .}
\end{gathered}
$$

However, $2 i_{s}+\left|x_{s-1}\right| \equiv 0\left(p^{t_{m}+1}\right)\left[i_{s}+\left|x_{s-1}\right| \equiv 0\left(2^{t_{m}+1}\right)\right]$ by Lemma 2.7 , so that forces $r=t_{m}$, and thus the lemma holds if $\phi(m)=s$.

If $\phi(m)>s$ then $i_{s-1}-i_{s}=u_{j} p^{t_{j}}+\cdots+u_{m-1} p^{t_{m-1}}$, so $2 i_{s}+\left|x_{s-1}\right|=2 v p^{r}$ $\left[i_{s}+\left|x_{s-1}\right|=v 2^{r}\right]$. Now $i_{s}=i_{s+1}=\cdots=i_{\phi(m)-1}$, and so

$$
\begin{gathered}
2 i_{\phi(m)-1}+\left|x_{\phi(m)-2}\right|=p^{\phi(m)-1-s}\left(2 i_{s}+\left|x_{s-1}\right|\right)=2 v p^{\phi(m)-1-s+r} \\
{\left[i_{\phi(m)-1}+\left|x_{\phi(m)-2}\right|=2^{\phi(m)-1-s}\left(i_{s}+\left|x_{s-1}\right|\right)=v 2^{\phi(m)-1-s+r}\right] .}
\end{gathered}
$$

Since

$$
\begin{gathered}
2 i_{\phi(m)}+\left|x_{\phi(m)-1}\right|=2\left(i_{\phi(m)}-i_{\phi(m)-1}\right)+p\left(2 i_{\phi(m)-1}+\left|x_{\phi(m)-2}\right|\right) \\
{\left[i_{\phi(m)}+\left|x_{\phi(m)-1}\right|=i_{\phi(m)}-i_{\phi(m)-1}+2\left(i_{\phi(m)-1}+\left|x_{\phi(m)-2}\right|\right)\right],}
\end{gathered}
$$

using Lemma 2.7 gives $0 \equiv 2 u_{m} p^{t_{m}}+2 v p^{\phi(m)-s+r}\left(\bmod p^{t_{m}+1}\right)\left[0 \equiv 2^{t_{m}}+\right.$ $\left.v 2^{\phi(m)-s+r}\left(\bmod 2^{t_{m}+1}\right)\right]$, and this forces $t_{m}=\phi(m)-s+r$ so that $\phi(m)=s+t_{m}$ $-r$, as required.

Thus if $Q_{2(p-1) I}\left[Q_{I}\right] \in N_{n}[k]$, then $I$ is completely determined by $i_{1}$ and $i_{k}$.

LEMMA 2.10. Let $Q_{2(p-1) I}, Q_{2(p-1) J} \in N_{n}[k]\left[Q_{I}, Q_{J} \in N_{n}[k]\right]$ and suppose $\left|Q_{2(p-1) I}\right|=\left|Q_{2(p-1) J}\right|\left[\left|Q_{I}\right|=\left|Q_{J}\right|\right]$. Then $I=J$.

Proof. Assume by induction that $i_{l}=j_{l}$ for $l<s$. (To begin the induction, $i_{0}=j_{0}=0$ by convention.) Let $x_{k}=Q_{2(p-1) I}\left[Q_{I}\right]$ and $y_{k}=Q_{2(p-1) J}\left[Q_{J}\right]$. Suppose $2 i_{s}+\left|x_{s-1}\right| \equiv u p^{t}\left(\bmod p^{t+1}\right), 1 \leqslant u<p\left[i_{s}+\left|x_{s-1}\right| \equiv 2^{t}\left(\bmod 2^{t+1}\right)\right]$. By Lemma $2.5, i_{l}-i_{l+1} \equiv 0\left(p^{t+1}\right)$ for all $l \geqslant s$ and $2 i_{l}+\left|x_{l-1}\right| \equiv 0\left(p^{t}\right)\left[i_{l}+\left|x_{l-1}\right| \equiv 0\left(2^{t}\right)\right]$ for all $l \geqslant s$. In particular, $i_{s} \equiv i_{s+1} \equiv \cdots \equiv i_{k}\left(\bmod p^{t+1}\right)$. Therefore,

$$
\begin{gathered}
\left|Q_{2(p-1) I}\right|=\left|x_{k}\right|=-2 i_{k}+p\left(2 i_{k}+\left|x_{k-1}\right|\right) \equiv-2 i_{k} \equiv-2 i_{s} \quad\left(\bmod p^{t+1}\right) \\
{\left[\left|Q_{I}\right|=\left|x_{k}\right|=-i_{k}+2\left(i_{k}+\left|x_{k-1}\right|\right) \equiv-i_{k} \equiv-i_{s} \quad\left(\bmod 2^{t+1}\right)\right] .}
\end{gathered}
$$


So $\left|x_{s-1}\right|-\left|x_{k}\right| \equiv u p^{t}\left(\bmod p^{t+1}\right)$. Similarly, if $2 j_{s}+\left|y_{s-1}\right| \equiv v p^{r}\left(\bmod p^{r+1}\right), 1 \leqslant v$ $<p\left[j_{s}+\left|y_{s-1}\right| \equiv 2^{r}\left(\bmod 2^{r+1}\right)\right]$, then $\left|y_{s-1}\right|-\left|y_{k}\right| \equiv v p^{r}\left(\bmod p^{r+1}\right)$. However, $\left|x_{k}\right|=\left|y_{k}\right|$ and $x_{s-1}=y_{s-1}$ since $i_{l}=j_{l}$ for $l<s$, and so we get $r=t$ and $u p^{t}=v p^{t}$. Thus

$$
\begin{gathered}
i_{s} \equiv-\frac{1}{2}\left|Q_{2(p-1) I}\right| \equiv-\frac{1}{2}\left|Q_{2(p-1) J}\right| \equiv j_{s} \quad\left(\bmod p^{t+1}\right) \\
{\left[i_{s} \equiv-\left|Q_{I}\right| \equiv-\left|Q_{J}\right| \equiv j_{s} \quad\left(\bmod 2^{t+1}\right)\right] .}
\end{gathered}
$$

Thus if $i_{s}<j_{s}$ then $i_{s}+p^{t+1} \leqslant j_{s} \leqslant n$, so by definition of $N_{n}[k], 2 i_{s}+\left|x_{s-1}\right| \equiv$ $0\left(p^{t+2}\right)\left[i_{s}+\left|x_{s-1}\right| \equiv 0\left(2^{t+2}\right)\right]$, which is a contradiction. Similarly, $j_{s}<i_{s}$ is impossible, so $j_{s}=i_{s}$.

Theorem 2.5 follows immediately from

TheOrem 2.11. Let $x \in A_{*} V$. Then when $x$ is written in the basis of admissible sequences, no terms in $N_{n}[k]$ occur.

Proof. It suffices to show that if $Q_{2(p-1) I}\left[Q_{I}\right] \in N_{n}[k]$ then

(a) $Q_{2(p-1) I}\left[Q_{I}\right]$ does not occur in any nontrivial Nishida relation (the trivial relation being the one for $P_{*}^{0}$ ), and

(b) $Q_{2(p-1) I}\left[Q_{I}\right]$ does not occur in any Adem relation.

To show (a): If there exists an $m>0$ and $Q_{2(p-1) J}\left[Q_{J}\right] \in V$ such that $P_{*}^{m} Q_{2(p-1) J}$ $\left[\mathrm{Sq}_{*}^{m} Q_{J}\right]$ has a $Q_{2(p-1) I}\left[Q_{I}\right]$ term after applying the Nishida relations but before rewriting with the Adem relations, then $P_{*}^{r} Q_{2(p-1)\left(i_{s}+r\right)}=Q_{2(p-1) i_{s}}\left[\mathrm{Sq}_{*}^{r} Q_{i_{s}+r}=Q_{i_{s}}\right]$ for some $s$ and some $r$, so

$$
\left(\begin{array}{c}
\left(2 i_{s}+\left|x_{s-1}\right|\right)((p-1) / 2) \\
r
\end{array}\right) \neq 0 \quad\left[\left(\begin{array}{c}
\left.i_{s}+\left|x_{s-1}\right|\right) \neq 0 \\
r
\end{array}\right) .\right.
$$

Suppose $\nu_{p}\left(2 i_{s}+\left|x_{s-1}\right|\right)=t\left[\nu_{2}\left(i_{s}+\left|x_{s-1}\right|\right)=t\right]$. Then the nonzero binomial coefficient implies that $r \equiv 0\left(p^{t}\right)$, so $r \geqslant p^{t}$. But then since $Q_{2(p-1) j_{s}}=Q_{2(p-1)\left(i_{s}+r\right)} \in V$ $\left[Q_{j_{s}}=Q_{i_{s}+r} \in V\right], i_{s}+p^{t} \leqslant i_{s}+r \leqslant j_{s} \leqslant n$, and so, by definition of $N_{n}[k], 2 i_{s}+$ $\left|x_{s-1}\right| \equiv 0\left(p^{t+1}\right)\left[i_{s}+\left|x_{s-1}\right| \equiv 0\left(2^{t+1}\right)\right]$, contradicting the definition of $t$.

To show (b):

$$
\begin{gathered}
Q_{2(p-1) a} Q_{2(p-1) b}=\sum_{j}(-1)^{a+(p-1) b+j}\left(\begin{array}{c}
(j-b)(p-1)-1 \\
a-j-1
\end{array}\right) \\
\cdot Q_{2(p-1)(a+p b-p j)} Q_{2(p-1) j} \\
{\left[Q_{a} Q_{b}=\sum_{j}\left(\begin{array}{c}
j-b-1 \\
a-j-1
\end{array}\right) Q_{a+2 b-2 j} Q_{j}\right] .}
\end{gathered}
$$

Suppose that $j=i_{s-1}$ and $a+p b-p j+i_{s}$ for some $a, b$ and $j$. From the binomial coefficient, $a-j \neq 0$, and so we can let $\nu_{p}(a-j)=t$. Then for a nonzero binomial coefficient, $j-b \equiv 0\left(p^{t}\right)$. By definition of $t, i_{s-1}+p^{t}=j+p^{t} \leqslant a \leqslant n$, and so, since $Q_{2(p-1) I}\left[Q_{I}\right] \in N_{n}[k], 2 i_{s-1}+\left|x_{s-2}\right| \equiv 0\left(p^{t+1}\right)\left[i_{s-1}+\left|x_{s-2}\right| \equiv 0\left(2^{t+1}\right)\right]$, and thus $i_{s-1}-i_{s} \equiv 0\left(p^{t+2}\right)$ by Lemma 2.5. Thus

$$
0 \equiv i_{s-1}-i_{s}=j-(a+p b-p j)=(j-a)+p(b-j) \quad\left(\bmod p^{t+2}\right)
$$

and so $a-j \equiv 0\left(p^{t+1}\right)$, contradicting the definition of $t$. 
3. Self-maps of $\Omega_{0}^{m+1} S^{m+1}$. Throughout the next three sections we will let $f$ denote a self-map of $\Omega_{0}^{m+1} S^{m+1}$ which satisfies Hypothesis 3.1. In this section we derive some homological properties of $f$ which are common to both the infinite loop case and the finite loop case.

Let $B$ denote the basis of monomials for $H_{*}\left(\Omega_{0}^{m+1} S^{m+1} ; Z / p Z\right)$ coming from its description as a free commutative graded algebra.

Hypothesis 3.1. (A) If $p=2, m \neq 1,3$ or 7. If $p>2, m$ is even or $m=\infty$.

(B) $f$ is an $H$-map.

(C) $f$ induces an isomorphism on $H_{2 p-3}\left(\Omega_{0}^{m+1} S^{m+1} ; Z / p Z\right)$.

In addition, if $p>2$,

(D) When written in the basis $B$,

$$
f_{*}\left(Q_{2(p-1)} Q_{2(p-1)}[1] *\left[-p^{2}\right]\right)=u Q_{2(p-1)} Q_{2(p-1)}[1] *\left[-p^{2}\right]+\text { other terms }
$$

for some unit $u$ in $Z / p Z$.

Theorem 3.3 will show that the additional Hypothesis 3.1(D) for $p>2$ is always satisfied if $f$ is a loop map satisfying the rest of Hypothesis 3.1.

REMARK. If $p=2$ the statement corresponding to Hypothesis 3.1(D) follows from Hypothesis 3.1(A)-(C). (See [S1, Lemma 5].) Conceivably, Hypothesis 3.1(D) may be redundant for $p>2$ also and, in fact, we conjecture that it is. If $m=\infty$, this would imply that the homotopy equivalence $Q_{0} S^{0} \approx S F(\approx \operatorname{Im} J \times \operatorname{Coker} J)$ is not an equivalence of $H$-spaces which at odd primes is not known, although $S F \approx \operatorname{Im} J$ $\times$ Coker $J$ is an infinite loop space splitting.

Replacing $f$ by $f^{p-1}$, we may suppose $f$ induces the identity on

$$
H_{2 p-3}\left(\Omega_{0}^{m+1} S^{m+1} ; Z / p Z\right)
$$

and that $u=1$ in Hypothesis 3.1(D).

THEOREM 3.2. Let $g$ be a self-map of $\Omega^{k} S^{k} S^{t}, 0 \leqslant k \leqslant \infty, t \geqslant 2$, which induces an isomorphism on $H_{t}\left(\Omega^{k} S^{m+1} ; Z / p Z\right)$, where $m=k+t-1$. Suppose that $m$ is even or $m=\infty$ if $p>2$, or that $m \neq 1,3$ or 7 if $p=2$. Let $f=\Omega^{t} g: \Omega_{0}^{m+1} S^{m+1} \rightarrow \Omega_{0}^{m+1} S^{m+1}$. Then $f$ satisfies Hypothesis 3.1 .

Proof. We will give the argument for $p>2$. The proof for $p=2$ is analogous and is given in [S1, Corollary 4].

Let $q=\Omega^{t-2} g$. Any self-map of $\Omega^{m-1} S^{m-1} S^{2}$ which induces an isomorphism on $H^{2}\left(\Omega^{m-1} S^{m-1} S^{2} ; Z_{(p)}\right)$ also induces an isomorphism on $H^{2 p}\left(\Omega^{m-1} S^{m-1} S^{2} ; Z_{(p)}\right)$, so reducing modulo $p$ and dualizing gives that $q$ induces an isomorphism on $H_{2 p}\left(\Omega^{m-1} S^{m-1} S^{2} ; Z / p Z\right)$. The homology suspension now shows that $f$ induces an isomorphism on $H_{2 p-2}\left(\Omega^{m+1} S^{m+1} ; Z / p Z\right)$, and so applying $\beta_{*}$ gives that $f_{*}$ is an isomorphism on $H_{2 p-3}\left(\Omega^{m+1} S^{m+1} ; Z / p Z\right)$. The rest will follow from Theorem 3.3.

THEOREM 3.3. Let $g$ be a self-map of $\Omega_{0}^{m+1} S^{m+1}$ which induces an isomorphism on $H_{2 p-3}\left(\Omega_{0}^{m+1} S^{m+1} ; Z / p Z\right)$ where if $p>2, m$ is even or $m=\infty$, and if $p=2, m \neq 1$, 3 or 7 . Suppose that $g$ is a loop map if $p>2$ or that $g$ is an $H$-map if $p=2$. Then when 
written in the basis $B$,

$$
\begin{gathered}
g_{*}\left(Q_{2(p-1)} Q_{2(p-1)}[1] *\left[-p^{2}\right]\right)=u Q_{2(p-1)} Q_{2(p-1)}[1] *\left[-p^{2}\right]+\text { other terms } \\
{\left[g_{*}\left(Q_{1} Q_{1}[1] *[-4]\right)=Q_{1} Q_{1}[1] *[-4]+\text { other terms }\right]}
\end{gathered}
$$

where $u$ is a unit in $Z / p Z$.

Proof. We will give the argument for $p>2$. For $p=2$ the proof is given in [S1, Lemma 5], although if we were willing to assume that $g$ is a loop map, an analogous proof to the one given below would be sufficient. We shall use elements of $R_{n}$ to denote their images under $\Phi: R_{n} \rightarrow H_{*}\left(\Omega_{0}^{m+1} S^{m+1} ; Z / p Z\right)$.

Let $g=\Omega q$ for some $q: \widetilde{\Omega^{m} S^{m} S^{1}} \rightarrow \widetilde{\Omega^{m} S^{m} S^{1}}$ where $\tilde{X}$ den0tes the universal covering space of $X$. Let $x=\sigma_{*}[1] \in H_{1}\left(\Omega^{m} S^{m} S^{1} ; Z / p Z\right)$ where $\sigma_{*}$ is the homology suspension, and den0te elements of $H_{*}\left(\Omega^{m} S^{m} S^{1} ; Z / p Z\right)$ by their images under the inclusion into $H_{*}\left(\Omega^{m} S^{m} S^{1} ; Z / p Z\right)$. In lower $Q$ notation, the commutativity of $\sigma_{*}$ with the Dyer-Lashof operations is expressed by $\sigma_{*} Q_{j(p-1)} y=Q_{(j-1)(p-1)} \sigma_{*} y$.

By hypothesis, $g_{*} \beta_{*} Q_{2(p-1)}=u \beta_{*} Q_{2(p-1)}$, and so $g_{*} Q_{2(p-1)}=u Q_{2(p-1)}$. Applying $\sigma_{*}$ and then $\beta_{*}$ gives $q_{*}\left(Q_{p-1} x\right)=u Q_{p-1} x$ and $q_{*}\left(\beta_{*} Q_{p-1} x\right)=$ $u \beta_{*} Q_{p-1} x$. Upon considering the commutativity of $q_{*}$ with the coalgebra structure, we find

$$
\begin{array}{rl}
q_{*}\left(Q_{p-1} x *\left(\beta_{*} Q_{p-1} x\right)^{p-1}\right) \equiv u Q_{p-1} x & *\left(\beta_{*} Q_{p-1} x\right)^{p-1} \\
& \bmod P H_{2 p^{2}-2 p+1}\left(\Omega^{m} S^{m} S^{1} ; Z / p Z\right) .
\end{array}
$$

Thus

$$
q_{*}\left(Q_{p-1} x *\left(\beta_{*} Q_{p-1} x\right)^{p-1}\right)=u Q_{p-1} x *\left(\beta_{*} Q_{p-1} x\right)^{p-1}+\lambda Q_{(2 p-1)(p-1)} x
$$

for some $\lambda \in Z / p Z$. Applying $\beta_{*}$ gives

$$
q_{*}\left(\left(\beta_{*} Q_{p-1} x\right)^{p}\right)=u\left(\beta_{*} Q_{p-1} x\right)^{p}+\lambda \beta_{*} Q_{(2 p-1)(p-1)} x .
$$

This implies

(4) $g_{*}\left(Q_{p-1} \beta_{*} Q_{2(p-1)}\right)=u Q_{p-1} \beta_{*} Q_{2(p-1)}+\lambda \beta_{*} Q_{2 p(p-1)}+$ decomposables.

The Nishida relations show that $P_{*}^{1} \beta_{*} Q_{2(p-1)} Q_{2(p-1)}=-Q_{p-1} \beta_{*} Q_{2(p-1)}$ and there is no other element of $B$ whose image under $P_{*}^{1}$ contains $Q_{p-1} \beta_{*} Q_{2(p-1)}$ when written in the basis $B$, so (4) implies

$$
g_{*} Q_{2(p-1)} Q_{2(p-1)}=u Q_{2(p-1)} Q_{2(p-1)}+\text { other terms. }
$$

Let $G=\left\{b \in B \mid f_{*}(b)=b+\right.$ other terms, when written in the basis $\left.B\right\}$.

THEOREM 3.4. Let $f$ satisfy Hypothesis 3.1. Then

$$
\left(Q_{2(p-1)}\right)^{s}[1] *\left[-p^{s}\right] \in G \quad\left[\left(Q_{1}\right)^{s}[1] *\left[-2^{s}\right] \in G\right],
$$

where $\left(Q_{j}\right)^{s}$ den 0 tes the s-fold iterate of the operation $Q_{j}$.

Proof. Let $h_{s}=\left(Q_{2(p-1)}\right)^{s}[1] *\left[-p^{s}\right]\left[h_{s}=\left(Q_{1}\right)^{s}[1] *\left[-2^{s}\right]\right]$. The proof is by induction on $s$ with $s=1$ given. When $s=2$ the statement is given for $p>2$ and has already been proved for $p=2$, so suppose the theorem is true for $s-1, s \geqslant 3$. 
Since $f$ is an $H$-map and $H_{*}\left(\Omega_{0}^{m+1} S^{m+1} ; Z / p Z\right)$ is a free commutative graded algebra, $\left(h_{s-1}\right)^{p} \in G$. The Nishida relations show that $P_{*}^{1} h_{s}=-\left(h_{s-1}\right)^{p}$, and since $s \geqslant 3$ there is no other element of $B$ whose image under $P^{1}{ }_{*}$ contains a nonzero multiple of $\left(h_{s-1}\right)^{p}$ when written in the basis $B$, so the result follows.

The composite

$$
\begin{aligned}
R_{n}[k] / Q_{0} R_{n}[k] & \mapsto \bigoplus_{k} R_{n}[k] / Q_{0} R_{n}[k] \\
& \cong Q H_{*}\left(\Omega_{0}^{m+1} S^{m-1} ; Z / p Z\right) \stackrel{f_{*}}{\rightarrow} Q H_{*}\left(\Omega_{0}^{m+1} S^{m+1} ; Z / p Z\right) \\
& \cong \bigoplus_{k} R_{n}[k] / Q_{0} R_{n}[k] \rightarrow R_{n}[k] / Q_{0} R_{n}[k]
\end{aligned}
$$

will be denoted $f[k]_{*}$. Its dual $f[k]^{*}$ can be defined similarly.

COROLlaRY 3.5. In $\left(R_{n}[k] / Q_{0} R_{n}[k]\right)^{*}, f[k]^{*}\left(a_{k k}\right)=a_{k k}$ for all $k$.

Proof. This is immediate from Theorem 3.4, the definition of $a_{k k}$, and the fact that there is no monomial $x$ in $\left(R_{n}[k] / Q_{0} R_{n}[k]\right)^{*}$ other than $a_{k k}$ such that $|x|=2\left(p^{k}-1\right)\left[|x|=2^{k}-1\right]$.

We conclude this section with the following observation:

LEMMA 3.6. In $\left(R_{n} / Q_{0} R_{n}\right)^{*}, f^{*}\left(W_{n}\right) \subset W_{n}$ where $W_{n}=\oplus W_{n}[k]$ as defined in $\S 2$ following the proof of Lemma 2.1 .

Proof. This is immediate from the equality $j_{n}^{*}(P[k])=\bigcap_{s=0}^{\infty} \operatorname{Ker} Q^{\Delta_{s}}$ of Corollary 1.8 .

4. The infinite loop case. In this section we prove our main theorem in the infinite loop case. The proof in this case is relatively straightforward in comparison to that of its analogue in the finite loop case.

THEOREM 4.1. Let $f: \Omega_{0}^{\infty} S^{\infty} \rightarrow \Omega_{0}^{\infty} S^{\infty}$ be an $H$-map which induces an isomorphism on $H_{2 p-3}\left(\Omega_{0}^{\infty} S^{\infty} ; Z / p Z\right)$. If $p>2$ suppose in addition that $f$ is a loop map or that, when written in the basis $B$,

$$
f_{*}\left(Q_{2(p-1)} Q_{2(p-1)}[1] *\left[-p^{2}\right]\right)=u Q_{2(p-1)} Q_{2(p-1)}[1] *\left[-p^{2}\right]
$$

for some unit $u$ in $Z / p Z$. Then $f_{(p)}$ is a homotopy equivalence.

COROLlary 4.2. Let $g$ be a self-map of $\Omega^{\infty} S^{\infty} S^{t}, t \geqslant 2$, such that $g$ induces an isomorphism on $H_{t}\left(\Omega^{\infty} S^{\infty} S^{t} ; Z / p Z\right)$. Then $g_{(p)}$ is a homotopy equivalence.

Proof of TheOrem 4.1. It suffices to show that $f$ induces an isomorphism on $P H^{*}\left(\Omega_{0}^{\infty} S^{\infty} ; Z / p Z\right)$ or, equivalently, that it induces a monomorphism, since $P H^{*}\left(\Omega_{0}^{\infty} S^{\infty} ; Z / p Z\right)$ has finite type. By Corollary $3.5, f[k]^{*}\left(a_{k k}\right)=a_{k k}$ for all $k$. Although in general $f^{*}$ need not commute with the multiplication operation in $\left(R / Q_{0} R\right)^{*}$ (see the remark above the discussion preceding Theorem 1.1), it does commute with the $p^{t}$ th power operation since the $p^{t}$ th power operation is a Steenrod operation. Thus $f[k]^{*}\left(a_{k k}^{p^{t}}\right)=a_{k k}^{p^{t}}$ for all $k$ and $t$, and so in particular $f^{*}\left(a_{k k}^{p^{t}}\right) \neq 0$. We will show that $f^{*}$ is a monomorphism, completing the proof, by showing in Lemmas 4.3 and 4.4 that, given any nonzero $x \in P H^{*}\left(\Omega_{0}^{\infty} S^{\infty} ; Z / p Z\right)$ such that $x \in \operatorname{Ker} f^{*}$, then $a_{k k}^{p^{t}} \in \operatorname{Ker} f^{*}$ for some $k$ and $t$. 
For $x \in\left(R / Q_{0} R\right)^{*} \cong \oplus_{k}\left(R[k] / Q_{0} R[k]\right)^{*}$, we write $x=\left(x_{j}\right)$ where $x_{j}$ is the $j$ th component of $x$. Recall that if $x \in P=\oplus_{k} P[k], d\left(x_{j}\right)$ denotes the degree of the polynomial $x_{j}$.

LEMma 4.3. Let $x \in\left(R / Q_{0} R\right)^{*}$ be nonzero. Then there exists a nonzero $y$ such that $y=\alpha x$ for some Steenrod operation $\alpha$ and $y \in P$.

Proof. Suppose $k$ is maximum such that $x_{k} \neq 0$. Set $x^{(0)}=x$ and inductively define

$$
x^{(t)}=\left\{\begin{array}{ll}
Q^{\Delta_{t-1}} x^{(t-1)} & \text { if } Q^{\Delta_{t-1}} x^{(t-1)} \neq 0 \\
x^{(t-1)} & \text { if } Q^{\Delta_{t-1}} x^{(t-1)}=0
\end{array}\right\} .
$$

Let $y=x^{(k)}$. By Corollary 1.5, $y \in P$, and the other properties are obvious.

Let $x=\left(x_{j}\right) \in P$ be nonzero and let $k$ be maximum such that $x_{k} \neq 0$. We will define an operation $\theta$ such that $\theta(x)$ has the following properties:

(1) $\theta(x) \neq 0$,

(2) $\theta(x)$ is a $p$ th power,

(3) for all $j, d\left((\theta(x))_{j}\right) \leqslant$ least integer divisible by $p$ which is greater than or equal to $d\left(x_{j}\right)$, and further, if $d\left((\theta(x))_{j}\right)>d\left(x_{j}\right)$ for some $j$ then $(\theta(x))_{i}=0$ for $i<j$.

To construct $\theta$ set $x^{(0)}=x$ and inductively define

$$
x^{(t)}=\left(\left(P^{\Delta_{t}}\right)^{s_{t}}\right) x^{(t-1)},
$$

where $s_{t} \geqslant 0$ is maximum, such that $\left(\left(P^{\Delta_{t}}\right)^{s_{t}}\right) x^{(t-1)} \neq 0$. Set $\theta=\prod_{t=1}^{k}\left(P^{\Delta_{t}}\right)^{s_{t}}$, so that $\theta(x)=x^{(k)}$. By construction, $\theta(x)$ is nonzero and $\theta(x) \in \bigcap_{j=1}^{k} \operatorname{Ker} P^{\Delta_{j}}$, so $\theta(x)$ is a $p$ th power by Corollary 1.4. In fact, for $j \leqslant k$, the first $j-1$ components of $x^{(j-1)}$ will be $p$ th powers. Since $P^{\Delta_{j}}$ kills $p$ th powers and the degree of the $j$ th component can increase only when $P^{\Delta_{j}}$ is applied, the third property follows.

Write $\theta(x)=y^{p}$ and set $\theta_{1}=\theta$. Applying the above procedure to $y$ gives an operation $\sigma$ such that $\sigma(y)$ is a nonzero $p$ th power with property (3) above. This allows us to construct an operation $\theta_{2}$ which converts $x$ to a $p^{2}$ th power by setting $\theta_{2}=(p \sigma) \circ \theta_{1}$ so that $\theta_{2}(x)=(\sigma(y))^{p}$, where $p \sigma$ is the operation obtained by multiplying each entry of each term of $\sigma$ by $p$.

Continuing, we inductively define operations $\theta_{t}$ such that $\theta_{t}$ has the properties:

(1) $\theta_{t}(x) \neq 0$,

(2) $\theta_{t}(x)$ is a $p^{t}$ th power,

(3) for all $j, d\left(\left(\theta_{t}(x)\right)_{j}\right) \leqslant$ least integer divisible by $p^{t}$ which is greater than or equal to $d\left(x_{j}\right)$, and further, if $d\left(\theta_{t}(x)_{j}\right)>d\left(x_{j}\right)$ for some $j$ then $\left(\theta_{t}(x)\right)_{i}=0$ for $i<j$.

LeMma 4.4. Let $x \in P$ be nonzero. Then for sufficiently large $t, \theta_{t}(x)=a_{k k}^{p^{t}}$ for some $k$.

Proof. Choose $r$ such that $p^{r}>\max \left\{d\left(x_{j}\right)\right\}$ and let $k$ be maximum such that $\left(\theta_{r}(x)\right)_{k} \neq 0$. Since $d\left(\left(\theta_{r}(x)\right)_{k}\right)$ is nonzero and divisible by $p^{r}, d\left(\left(\theta_{r}(x)\right)_{k}\right)>d\left(x_{k}\right)$, and so all the components of $\theta_{r}(x)$ except for the $k$ th are zero. Choose $s$ such that, for each $j<k, p^{s}$ is greater than the exponent of $a_{j k}$ in any monomial of $\theta_{r}(x)$. Then 
$\theta_{s}(x)=a_{k k}^{\lambda p^{s}}$ since $\theta_{s}(x)$ is a $p^{s}$ th power and the process of forming $\theta_{s}(x)$ from $\theta_{r}(x)$ cannot increase the exponent of $a_{j k}$ for $j<k$. Find $v$ such that $\lambda p^{s}<p^{v}$. Then $\theta_{t}(x)=a_{k k}^{p^{t}}$ for all $t \geqslant v$.

Proof of Corollary 4.2. This follows immediately from Theorems 3.2 and 4.1.

5. The finite loop case. In this section we prove our main theorem in the finite loop case.

THEOREM 5.1. Let $f: \Omega_{0}^{m+1} S^{m+1} \rightarrow \Omega_{0}^{m+1} S^{m+1}$ be an $H$-map which induces an isomorphism on $\mathrm{H}_{2 p-3}\left(\Omega_{0}^{m+1} S^{m+1} ; Z / p Z\right)$. Suppose $m<\infty$. If $p>2$ suppose in addition that $m$ is even and that either $f$ is a loop map or that, when written in the basis $B$,

$$
f_{*}\left(Q_{2(p-1)} Q_{2(p-1)}[1] *\left[-p^{2}\right]\right)=u Q_{2(p-1)} Q_{2(p-1)}[1] *\left[-p^{2}\right]+\text { other terms }
$$

for some unit $u \in Z / p Z$. If $p=2$ we suppose $m \neq 1,3$ or 7 . Then $f_{(p)}$ is a homotopy equivalence.

COROLlaRY 5.2. Let $g$ be a self-map of $\Omega^{k} S^{m+1}, k<m<\infty$, which induces an isomorphism on $H_{m+1-k}\left(\Omega^{k} S^{m+1} ; Z / p Z\right)$. If $p>2$ we suppose also that $m$ is even, and if $p=2$ we suppose $m \neq 1,3$ or 7 . Then $g_{(p)}$ is a homotopy equivalence.

Let $f$ satisfy Hypothesis 3.1 with $m<\infty$. The proof of Theorem 5.1 is considerably more complicated than that of its infinite loop analogue, Theorem 4.1. The argument of $\$ 4$ fails because $\left(a_{k k}\right)^{p^{t}}$ will be zero for large $t$. Instead we take advantage of the fact that, since $m<\infty,\left(R_{n}[k] / Q_{0} R_{n}[k]\right)^{*}$ is finite dimensional for each $k$ (not just finite type), and thus if there is any nonzero

$$
x \in P H^{*}\left(\Omega_{0}^{m+1} S^{m+1} ; Z / p Z\right)
$$

which lies in $\operatorname{Ker} f^{*}$ then there is a nonzero $x \in \operatorname{Ann} P H^{*}\left(\Omega_{0}^{m+1} S^{m+1} ; Z / p Z\right)$ which lies in $\operatorname{Ker} f^{*}$. By Lemma 2.1, if $x \in \operatorname{Ann} P H^{*}\left(\Omega_{0}^{m+1} S^{m+1} ; Z / p Z\right)$ then $x \in \operatorname{Ann}\left(R_{n}[k] / Q_{0} R_{n}[k]\right) *$ for some $k$. Since we have computed

$$
\operatorname{Ann}\left(R_{n}[k] / Q_{0} R_{n}[k]\right)^{*}
$$

in $\S 2$, the obvious approach might be to attempt to check directly that $f^{*}(x) \neq 0$ for each $x \in \operatorname{Ann}\left(R_{n}[k] / Q_{0} R_{n}[k]\right)^{*}$, but it was not clear to us how to do this in general. Instead, we argue independently of $\$ 2$ that, for each $k, f[k]^{*}(x) \neq 0$ for any $x$ belonging to the principal ideal $\left(a_{k k}^{p}\right)$. Finally, we make use of the calculation of $\$ 2$ to check that $f[k]^{*}(x) \neq 0$ for the elements $\operatorname{Ann}\left(R_{n}[k] / Q_{0} R_{n}[k]\right)^{*}$ lying outside of this ideal.

Since $k$ is fixed we will again write simply $a_{j}$ for $a_{j k}$. By Lemma $3.6 f[k]^{*}$ restricts to a self-map of $\left(a_{k}\right)$ where here as always $\left(a_{k}\right)$ refers to the principal ideal in $P[k]=Z / p Z\left[a_{1}, \ldots, a_{k}\right]$ generated by $a_{k}$. Let $K=\bigcap_{j=1}^{k-1} \operatorname{Ker} P^{\Delta_{j}} \cap\left(a_{k}\right)$.

Our immediate goal is the proof of the following two technical theorems which we will use in the proof of Theorem 5.1. 
THEOREM 5.3. There exists an integer $N$ (depending on $k$ ) such that $\left(f[k]^{*}\right)^{N}(x)=x$ for all polynomials $x \in\left(a_{k}\right)$ such that $d(x) \leqslant(n+1) / p$. Furthermore, if $n \equiv 0(p)$, the restriction $d(x) \leqslant(n+1) / p$ can be removed.

THEOREM 5.4. Suppose $n>p$. There exists an integer $N$ (depending on $k$ ) such that $\left(f[k]^{*}\right)^{N}(x)=x$ for all polynomials $x \in K \cap\left(a_{k}^{p}\right)$.

If $z \in K$, the exponent of $a_{j}$ for $j<k$ is divisible by $p$ in each monomial of $z$ so each monomial of $z$ lies in $K$. For a monomial $a=a_{1}^{r_{1}} a_{2}^{r_{2}} \cdots a_{k}^{r_{k}},|a| \equiv-2 r_{k}$ $(\bmod p)\left[|a| \equiv r_{k}(\bmod 2)\right]$, and thus if $a \in K, d(a) \equiv r_{k} \equiv-\frac{1}{2}|a|(\bmod p)[d(a) \equiv$ $\left.r_{k} \equiv|a|(\bmod 2)\right]$.

Lemma 5.5. Let $x \in\left(a_{k}\right)$ and suppose $d(x) \leqslant s$ for $s \geqslant 2$, where $d(x)$ denotes the degree of the polynomial $x$. Then $d\left(f[k]^{*}(x)\right) \leqslant s p-p$.

Proof. Since $d(x) \leqslant s,|x| \leqslant\left|a_{k}^{s}\right|=2 s\left(p^{k}-1\right)\left[s\left(2^{k}-1\right)\right]$. However, $\left|f[k]^{*}(x)\right|$ $=|x|$, and if $d(y)>s p-p$ then

$$
\begin{gathered}
|y| \geqslant\left|a_{1}^{s p-p} a_{k}\right|=(s p-p) 2\left(p^{k}-p^{k-1}\right)+2\left(p^{k}-1\right) \\
{\left[(2 s-2)\left(2^{k}-2^{k-1}\right)+2^{k}-1\right] .}
\end{gathered}
$$

Since $2 s\left(p^{k}-1\right)<(s p-p) 2\left(p^{k}-p^{k-1}\right)+2\left(p^{k}-1\right)$, the result follows.

Proof of Theorem 5.3. We will write simply $f$ for $f[k]^{*}$ and let $g=1-f$. Let

$$
\begin{aligned}
\mathscr{F} & =\left\{x \in\left(a_{k}\right) \mid f^{p^{r}} x=x \text { for some } r\right\} \\
& =\left\{x \in\left(a_{k}\right) \mid g^{p^{r}} x=0 \text { for some } r\right\}=\left\{x \in\left(a_{k}\right) \mid g^{t} x=0 \text { for some } t\right\} .
\end{aligned}
$$

It is clear from the third description that if $g x \in \mathscr{F}$ then $x \in \mathscr{F}$. Since $\left(a_{k}\right)$ is finite dimensional when $n<\infty$, it suffices to show that if $d(x) \leqslant(n+1) / p$ then $x \in \mathscr{F}$. Let $V_{j}=\left\{x \in\left(a_{k}\right) \mid d(x) \leqslant j\right\}$. We wish to show $V_{s} \subset \mathscr{F}$ for $s \leqslant(n+1) / p$. $V_{1}$ consists of multiples of $a_{k}$, so we have $V_{1} \subset \mathscr{F}$ by Corollary 3.5. Suppose by induction that $V_{s-1} \subset \mathscr{F}$ for some $s \leqslant(n+1) / p$. Replacing $g$ by $g^{p^{M}}$ for some $M$, we may assume $V_{s-1} \subset \operatorname{Ker} g$. Since the $p$ th power operation is a Steenrod operation, it commutes with $g$. Thus $V_{p s-p} \cap\{p$ th powers $\} \subset \operatorname{Ker} g$. Note that if $d(x)<n$ or if $n \equiv 0(p)$ then $x$ is a $p$ th power if and only if $x \in \bigcap_{j=1}^{k} \operatorname{Ker} P^{\Delta_{j}}$ if and only if $x \in \bigcap_{j=1}^{\infty} \operatorname{Ker} P^{\Delta_{j}}$, just as in Corollary 1.4.

Step 1. $V_{s p-p} \cap K \subset \mathscr{F}$.

Proof. Let $x \in V_{s p-p} \cap K$. Since $V_{s-1} \cup\left(V_{s p-p} \cap\{p\right.$ th powers $\left.\}\right) \subset \mathscr{F}$, it suffices to consider only $x$ having no monomials in $V_{s-1} \cup\left(V_{s p-p} \cap\{p\right.$ th powers $\left.\}\right)$. Suppose $d(x) \equiv j(\bmod p), 1 \leqslant j<p .(j \neq 0$ since $x$ is not a $p$ th power.) Let $y=$ $\left(P^{\Delta_{k}}\right)^{p-j} x$. Thus

$$
y=\frac{(p-1) !}{(j-1) !} x a_{k}^{p-j}
$$

is a $p$ th power. Since $d(x) \leqslant p s-p$ and $d(x) \equiv j(\bmod p)$, we must have $d(x) \leqslant p s$ $-p-(p-j)$, and so $d(y)=d(x)+p-j \leqslant p s-p$. Thus $y \in V_{s p-p} \cap\{p$ th powers $\} \subset \mathscr{F}$. Replacing $g$ by $g^{p^{M}}$ for some $M$ we may assume $y \in \operatorname{Ker} g$. Thus 
$\left(P^{\Delta_{k}}\right)^{p-j} g x=0$. Since $x \in K, g x \in K$, and so $d(g x) \equiv-\frac{1}{2}|g x|=-\frac{1}{2}|x| \equiv d(x)$ $\equiv j(\bmod p)[d(g x) \equiv|g x|=|x| \equiv d(x) \equiv 1(\bmod 2)]$. So

$$
\left(P^{\Delta_{k}}\right)^{p-j} g x=\frac{(p-1) !}{(j-1) !}(g x) a_{k}^{p-j} .
$$

However, by Lemma 5.1, $d\left((g x) a_{k}^{p-j}\right) \leqslant s p-p+p-j \leqslant n+1-j \leqslant n$, so $(g x) a_{k}^{p^{-j}}$ is already admissible, and therefore $((p-1) ! /(j-1) !)(g x) a_{k}^{p-j}=0$ forces $g x=0$. Thus $x \in \mathscr{F}$.

Step 2. $V_{s} \subset \mathscr{F}$.

Proof. The element of $V_{s}$ of largest total degree is $a_{k}^{s}$ which is in $V_{s p-p} \cap K \subset \mathscr{F}$. Let $x \in V_{s}$ and suppose by downward induction on total degree that $y \in \mathscr{F}$ for all $y \in V_{s}$ such that $|y|>|x|$. Replacing $g$ by $g^{p^{M}}$ for some $M$ we may assume that $y \in \operatorname{Ker} g$ for all $y \in V_{s}$ such that $|y|>|x|$ and that $V_{s p-p} \cap K \subset \operatorname{Ker} g$. Since $P^{\Delta_{j}} x \in V_{s}$ for $1 \leqslant j \leqslant k-1, P^{\Delta_{j}} x \in \operatorname{Ker} g$, so $g x \in K$. By Lemma 5.5, $d(g x) \leqslant s p$ - $p$, so $g x \in V_{s p-p} \cap K \subset \mathscr{F}$. Thus $x \in \mathscr{F}$, completing the proof of Step 2 .

To prove the stronger statement when $n \equiv 0(p)$, follow the proof of Step 1 as far as the conclusion that $\left(P^{\Delta_{k}}\right)^{p-j} g x=0$. Then assume by downward induction on $i$ that $\left(P^{\Delta_{k}}\right) g x=0, j+1 \leqslant i \leqslant p-j$. So $\left(P^{\Delta_{k}}\right)^{i-1} g x \in \bigcap_{j=1}^{k} \operatorname{Ker} P^{\Delta_{j}}$ which says that $\left(P^{\Delta_{k}}\right)^{i-1} g x$ is a $p$ th power since $n \equiv 0(p)$. But $\left|\left(P^{\Delta_{k}}\right)^{i-1} g x\right| \equiv-2 j-2(i-1) \equiv \equiv$ $0(p)[|g x| \equiv 1(2)]$, so we must have $\left(P^{\Delta_{k}}\right)^{i-1} g x=0$, completing the induction. This shows $g x \in \mathscr{F}$ and so $x \in \mathscr{F}$, completing the proof of Step 1 . The proof of Step 2 requires no modification.

The proof of the main theorem will be by induction on $k$. The initial statement for the induction will be proved in Lemma 5.6 except for the case $p=2, n=2^{t}-1$, $t \geqslant 4$, in which case we will also need Lemmas 5.7 and 5.8.

LEMMA 5.6. $f[1]_{*}$ is the identity on $R_{n}[1] / Q_{0} R_{n}[1]$ except possibly in the case $p=2$, $n=2^{t}-1$, in which case it is the identity in degrees less than $n$.

Proof. Write simply $f$ for $f[1]_{*}$ and use elements of $R_{n}$ to den0te their images under $\Phi$ in $H_{*}\left(\Omega_{0}^{m+1} S^{m+1} ; Z / p Z\right)$.

First consider $p>2$. We know $f \beta Q_{2(p-1)}=\beta Q_{2(p-1)}$ and so $f Q_{2(p-1)}=Q_{2(p-1)}$. Suppose by induction that $f Q_{2 j(p-1)}=Q_{2 j(p-1)}$ for $j<s$. Then we also have $f \beta Q_{2 j(p-1)}=\beta Q_{2 j(p-1)}$ for $j<s . \quad P_{*}^{1} Q_{2 s(p-1)}=(s-1) Q_{2(s-1)(p-1)}$ and $P_{*}^{1} \beta Q_{2 s(p-1)}=s \beta Q_{2(s-1)(p-1)}$ so at least one of $Q_{2 s(p-1)}$ and $\beta Q_{2 s(p-1)}$ goes nontrivially under $P^{1}$ and thus must be fixed by $f$. But then the other is fixed as well.

If $p=2$, we know $f Q_{1}=Q_{1}$. Suppose by induction that $f Q_{j}=Q_{j}$ for $j<s$. $\mathrm{Sq}_{*}^{r} Q_{s}=\left(\begin{array}{c}s-r \\ r\end{array}\right) Q_{s-r}$, so unless $s=2^{t}-1$ for some $t$, there is a $\mathrm{Sq}_{*}^{r}$ acting nontrivially on $Q_{s}$, and we can thus conclude that $f Q_{s}=Q_{s}$. If $s=2^{t}-1$ then, unless $s=n, \mathrm{Sq}_{*}^{2} Q_{s+1} \neq 0$ and so $f Q_{s+1}=Q_{s+1}$. Applying $\mathrm{Sq}_{*}^{1}$ then gives $f Q_{s}=Q_{s}$.

Lemmas 5.7 and 5.8 do the additional maneuvering necessary to extend Lemma 5.6 to the case $p=2, n=2^{t}-1, t \geqslant 4$.

LEMMA 5.7. Let $p=2$ and $n=2^{t}-1$. Then $f$ induces an isomorphism on $H_{q}\left(\Omega_{0}^{n+1} S^{n+1} ; Z / 2 Z\right)$ for $q<n$. 
Proof. Since $H_{*}\left(\Omega_{0}^{n+1} S^{n+1} ; Z / 2 Z\right)$ is an integral domain it suffices to show that $f_{*}$ is an isomorphism on $Q H_{q}\left(\Omega_{0}^{n+1} S^{n+1} ; Z / 2 Z\right)$ or equivalently that $f^{*}$ is an isomorphism on $P H^{q}\left(\Omega_{0}^{n+1} S^{n+1} ; Z / 2 Z\right)$ for $q<n$. Suppose that there is a nonzero $x \in P H^{q}\left(\Omega_{0}^{n+1} S^{n+1} ; Z / 2 Z\right)$ such that $f^{*} x=0$. Let $x=\left(x_{j}\right)$. Since $|x|<n, d\left(x_{j}\right)$ $\leqslant 2^{t-1}$ for $j \geqslant 2$. Since $\left(R_{n}[1] / Q_{0} R_{n}[1]\right)^{*} \cong H^{*}\left(R P^{n} ; Z / 2 Z\right)$ as an $A$-module, either $x_{1}=0$ or by applying Steenrod operations we may replace $x$ by a new nonzero $x$ in $\operatorname{Ker} f^{*}$ such that $x_{1}=a_{11}^{2^{t}-2^{s}}$ for some $s, 1 \leqslant s \leqslant t-1$. We wish to apply Steenrod operations to $x$ to obtain $y$ with the following properties:

(1) $y \neq 0$,

(2) $d\left(y_{j}\right) \leqslant 2^{t-1}$ for $j \geqslant 2$,

(3) either $y_{j}=0$ for all $j \geqslant 2$ or $y_{1}=0$.

To find $y$, first form $\theta_{s}(x)$ where $\theta_{s}$ is as in $\S 4$. By the properties of $\theta_{s}$, $d\left(\left(\theta_{s}(x)\right)_{j}\right) \leqslant 2^{t-1}$ for $j \geqslant 2$ and $d\left(\left(\theta_{s}(x)\right)_{1}\right) \leqslant 2^{t}-2^{s}$, so, in particular, each component has degree less than $n$ and thus $\theta_{s}(x)$ is already written admissibly. Thus $\theta_{s}(x) \neq 0$. Write $\theta_{s}(x)=v^{2^{s}}$. If either $v_{j}=0$ for all $j \geqslant 2$ or $v_{1}=0$, we can set $y=\theta_{s}(x)$, so suppose $v_{1} \neq 0$ and $v_{j} \neq 0$ for some $j \geqslant 2$. Let $k$ be minimum such that $k \geqslant 2$ and $v_{k} \neq 0$. Since $v_{1}=a_{11}^{2^{t-s}-1},|v|$ is odd, so $v_{k}$ is not a square. Thus $\mathrm{Sq}^{\Delta_{i}} v_{k} \neq 0$ for some $i \leqslant k$. Let $y=\left(\mathrm{Sq}^{\Delta_{i}}\right)^{2^{s}} \theta_{s}(x)$. Then $y_{1}=0, y_{k} \neq 0$, and using the minimality of $k, d\left(y_{j}\right) \leqslant 2^{t-1}$ for all $j$.

Having found $y$ with the three properties above, let

$$
w=\left\{\begin{array}{ll}
y & \text { if } y_{1} \neq 0, y_{j}=0, j \geqslant 2 \\
\theta_{t-1}(y) & \text { if } y_{1}=0, y_{j} \neq 0, j \geqslant 2
\end{array}\right\} .
$$

In the latter case, where $w=\theta_{t-1}(y)$, for each $j$ such that $y_{j} \neq 0, w_{j}$ is a $2^{t-1}$ th power such that $d\left(w_{j}\right) \leqslant 2^{t-1}$. Since $w_{j} \in\left(a_{j j}\right)$, the only possibilities are $w_{j}=0$ or $w_{j}=$ $\left(a_{j j}\right)^{2^{t-1}}$. Since $\left|a_{i i}\right| \neq\left|a_{j j}\right|$ for $i \neq j, w$ cannot have more than one nonzero component. By properties of $\theta_{t-1}, w \neq 0$, and so $w=\left(a_{k k}\right)^{2^{t-1}}$ for some $k$ in this case. Thus if there is a nonzero $x$ in $\operatorname{Ker} f^{*}$ with $|x|<n$, then either $a_{11}^{2^{t}-2^{s}} \in \operatorname{Ker} f^{*}$ for some $s, 1 \leqslant s \leqslant t-1$, or else $\left(a_{k k}\right)^{2^{t-1}} \in \operatorname{Ker} f^{*}$ for some $k$. However, $a_{11}^{2^{t}-2^{s}} \notin \operatorname{Ker} f^{*}$ by Lemma 5.6 and $\left(a_{k k}\right)^{2^{t-1}} \notin \operatorname{Ker} f^{*}$ by Theorem 5.3 (or in fact by Corollary 3.5). Thus $f^{*}$ is an isomorphism on $P H^{q}\left(\Omega_{0}^{n+1} S^{n+1} ; Z / 2 Z\right)$.

LEMma 5.8. Let $p=2$ and $n=2^{t}-1, t \geqslant 4$. Then $f[1]_{*}\left(Q_{n}[1] *[-2]\right)=$ $Q_{n}[1] *[-2]$.

Proof. Suppose $f[1]_{*}\left(Q_{n}[1] *[-2]\right)=0$. Since $a_{11}^{n}$ generates

$$
\text { Ann } P H^{n}\left(\Omega_{0}^{n+1} S^{n+1} ; Z / 2 Z\right),
$$

we must have $f^{*} a_{11}^{n}=\lambda a_{11}^{n}$ for some $\lambda \in Z / 2 Z$ and $f[1]_{*}\left(Q_{n}[1] *[-2]\right)=0$ implies that $\lambda=0$. It follows that there is no element of $H_{n}\left(\Omega_{0}^{n+1} S^{n+1} ; Z / 2 Z\right)$ lying in $\operatorname{Im} f_{*}$ which when written in the basis $B$ contains a nonzero $Q_{n}[1] *[-2]$ term. Let $V=f_{*}\left(H_{n}\left(\Omega_{0}^{n+1} S^{n+1} ; Z / 2 Z\right)\right)$. Since $H_{n}\left(\Omega_{0}^{n+1} S^{n+1} ; Z / 2 Z\right)$ is finite dimensional, by replacing $f$ by $f^{N}$ for sufficiently large $N$ we may assume that $f_{*} V=V$. Find $v \in V$ such that $f_{*} v=f_{*}\left(Q_{n}[1] *[-2]\right)$. Then $Q_{n}[1] *[-2]+v$ belongs to $\operatorname{Ker} f_{*}$ and when written in the basis $B$ the $Q_{n}[1] *[-2]$ term is not cancelled. Since $f_{*}$ is an 
isomorphism on $H_{q}\left(\Omega_{0}^{n+1} S^{n+1} ; Z / 2 Z\right)$ for $q<n$, it follows by considering the Hurewicz isomorphism in the homotopy-theoretic fibre of the canonical map from $\Omega_{0}^{n+1} S^{n+1}$ to the infinite mapping telescope $\lim _{f} \Omega_{0}^{n+1} S^{n+1}$ that any element of $H_{n}\left(\Omega_{0}^{n+1} S^{n+1} ; Z / 2 Z\right)$ which lies in $\operatorname{Ker} f_{*}$ is spherical. That is, there exists a map $g$ : $S^{2^{t}-1} \rightarrow \Omega_{0}^{2^{t}} S^{2^{t}}$ such that $g_{*}\left(\iota_{2^{t}-1}\right)=Q_{n}[1] *[-2]+v$. But upon applying the $\left(2^{t}-\right.$ 1)-fold iterate of the homology suspension, $v$ goes to zero and we get that $\left(\iota_{2^{t}-1}\right)^{2}$ is spherical in $H_{2^{t+1}-2}\left(\Omega S^{2^{t}} ; Z / 2 Z\right)$ which is equivalent to saying that the $2^{t}$-fold adjoint of $g$ has Hopf invariant one-a contradiction since $t \geqslant 4$.

The following lemma will be needed in the proof of Theorem 5.4. Recall the definition of $G$ from $\$ 3$.

LEMMA 5.9. Suppose $n>p$ and $n \not \equiv 0(p)$. Find s such that $n \equiv s(p)$ and $1 \leqslant s \leqslant p$. Then for $k \geqslant 0$

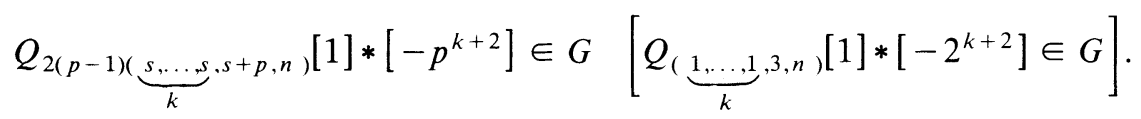

Proof. We will use elements of $R_{n}$ to denote their images under $\Phi$ in $H_{*}\left(\Omega_{0}^{n+1} S^{n+1}\right.$; $Z / \dot{p} Z$ ) and let $e_{k}$ denote

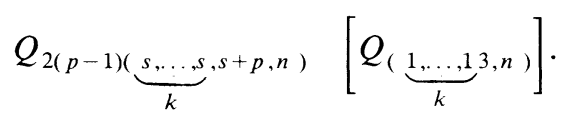

The proof is by induction on $k$.

To begin the induction we consider two cases. Write $n \equiv r p+s\left(\bmod p^{2}\right)$ where $0 \leqslant r<p$. Consider first the case $r \neq s$. Then computing with the Nishida relations gives

$$
P_{*}^{s} P_{*}^{p} e_{0}=(-1)^{s}(r-s)\left(Q_{2 n(p-1)}\right)^{p}\left[\left(Q_{n}\right)^{2}\right] .
$$

By Lemmas 5.6 and 5.8 the right-hand side of this equation is in $G$, using the fact that $f$ is an $H$-map. Since there is no other element of $B$ whose image under $P_{*}^{s} P_{*}^{p}$ contains a nonzero multiple of $\left(Q_{2 n(p-1)}\right)^{p}\left[\left(Q_{n}\right)^{2}\right]$ when written in $B$, this implies $e_{0} \in G$.

Next consider the case $r=s$. Then

$$
P_{*}^{s p} P_{*}^{s} e_{0}=(-1)^{s}\left(\begin{array}{c}
p-1 \\
s-1
\end{array}\right)\left(Q_{2(p-1)(n-s+1)}\right)^{p} \quad\left[\left(Q_{n}\right)^{2}\right]
$$

which is in $G$ as in the previous case. Once again there is no other element of $B$ whose image under $P_{*}^{s p} P_{*}^{p}$ contains a nonzero multiple of $\left(Q_{2(p-1)(n-s+1)}\right)^{p}\left[\left(Q_{n}\right)^{2}\right]$ when written in $B$. So $e_{0} \in G$ in this case also.

Proceeding to the general case, suppose we know $e_{k-1} \in G$ for some $k \geqslant 1$.

$$
P_{*}^{s} e_{k}=\left(e_{k-1}\right)^{p} \text {. }
$$

Since there is no element of $B$ whose image under $P_{*}^{1}$ contains a nonzero multiple of $e_{k-1}$ when written in the basis $B$, there is no element of $B$ other than $e_{k}$ whose image under $P_{*}^{s}$ contains $\left(e_{k-1}\right)^{p}$ when written in the basis $B$. Thus $e_{k} \in G$. 
Proof of Theorem 5.4. By Lemmas 5.6 and 5.8, it suffices to consider $k \geqslant 2$. We may assume $n \neq \equiv(p)$ since Theorem 5.3 contains a stronger result for $n \equiv 0(p)$. Let $f, g$ and $\mathscr{F}$ be as in the proof of Theorem 5.3. By Theorem 5.3 and Lemma 1.10, $\{p$ th powers $\} \subset \mathscr{F}$.

Given $x \in K$ with $d(x)=n$, let $u_{x}=1$ (admissible expression for $x a_{k}$ ) $/ a_{k}$. Then $u_{x} \in K$, and using Lemma 1.11, if $x$ is in $\left(a_{k}^{r}\right)$ so is $u_{x}$. Recall that for any monomial $a \in K, d(a) \equiv-\frac{1}{2}|a|(\bmod p)[d(a) \equiv|a|(\bmod 2)]$ and so the congruence classes of the degrees of monomials in $K$ are identical. Thus $d\left(u_{x}\right) \equiv d(x)(\bmod p)$. So

$$
P^{\Delta_{k}} u_{x}=d\left(u_{x}\right) u_{x} a_{k}=d(x) x a_{k}=P^{\Delta_{k}} x,
$$

and by construction $d\left(u_{x}\right)<n . u_{x}$ is characterized by the properties:

$$
\text { (1) } P^{\Delta_{k}}\left(x-u_{x}\right)=0 \text {, }
$$

$d\left(u_{x}\right)<n$,

(3) $u_{x} \in K$.

For if $v$ has these properties, then $v-u_{x} \in \bigcap_{j=1}^{k} \operatorname{Ker} P^{\Delta_{j}}$ and $d\left(v-u_{x}\right)<n$, so $v-u_{x}$ is a $p$ th power. But $\left|v-u_{x}\right|=|x| \equiv-2 d(x)=-2 n \not \equiv 0(p)\left[\left|v-u_{x}\right|=|x|\right.$ $\equiv d(x)=n \not 0(2)]$, so $v=u_{x}$.

For each congruence class modulo $p$, let

$$
K_{j}=\{x \in K \mid d(x) \equiv j(p)\}=\{x \in K|| x \mid \equiv-2 j\} \quad[\{x \in K|| x \mid=j\}]
$$

where $1 \leqslant j \leqslant p$. Note that for all $r \leqslant p, f\left(K_{j} \cap\left(a_{k}^{r}\right)\right) \subset K_{j} \cap\left(a_{k}^{r}\right)$ because $K_{j} \cap$ $\left(a_{k}^{r}\right)=K_{j} \cap \operatorname{Im}\left(P^{\Delta k-1}\right)^{r-1}$.

Find $s$ such that $1 \leqslant s \leqslant p-1$ and $n \equiv s(p) . K_{p}=\{p$ th powers $\}$ so $K_{p} \cap\left(a_{k}\right)^{p}$ $\subset \mathscr{F}$. Now let $t<p$ and suppose by downward induction on $t$ that $K_{i} \cap\left(a_{k}^{p}\right) \subset \mathscr{F}$ for $i>t$. Replacing $g$ by $g^{p^{M}}$ for some $M$ we may assume that $K_{i} \cap\left(a_{k}^{p}\right) \subset \operatorname{Ker} g$ for $i>t$. Let $x \in K_{t} \cap\left(a_{k}^{p}\right)$. Then $P^{\Delta_{k}} x \in K_{t+1} \cap\left(a_{k}^{p}\right) \subset \operatorname{Ker} g$ so $g x \in$ $\cap_{j=1}^{k} \operatorname{Ker} P^{\Delta_{j}}$. But if $y \in \bigcap_{j=1}^{k} \operatorname{Ker} P^{\Delta_{j}}$ then either $y$ is a $p$ th power or $d(y)=n$. Thus $\bigcap_{j=1}^{k} \operatorname{Ker} P^{\Delta_{j}} \subset K_{p} \cup K_{s}$, so if $t \neq s$ we must have $g x=0$, and so $x \in \mathscr{F}$. It remains to consider the case where $t=s$.

Let $\left(K_{s} \cap\left(a_{k}^{p}\right)\right)_{q}=\left\{x \in K_{s} \cap\left(a_{k}^{p}\right)|| x \mid=q\right\}$ and suppose by induction on $q$ that $\left(K_{s} \cap\left(a_{k}\right)^{p}\right)_{q} \subset \mathscr{F}$ for $q<R$. Replacing $g$ by $g^{p^{M}}$ for some $M$ we may assume that $\left(K_{s} \cap\left(a_{k}\right)^{p}\right)_{q} \subset \operatorname{Ker} g$ for $q<R$. We complete the induction by showing in three steps that $\left(K_{s} \cap\left(a_{k}^{p}\right)\right)_{R} \subset \mathscr{F}$. $\mathscr{F}$.

Step 1. Let $x$ be a monomial in $\left(K_{s} \cap\left(a_{k}^{p}\right)\right)_{R}$ such that $d(x)=n$. Then $x-u_{x} \in$

Proof. Either $x$ has the form $x=a_{1}^{p r_{1}} a_{j}^{p r_{j}} a_{j+1}^{p r_{j+1}} \cdots a_{k-1}^{p r_{k}-1} a_{k}^{p r_{k}} a_{k}^{p+s}$ where $r_{j} \geqslant 1$, $2 \leqslant j \leqslant k$, or else $x=a_{1}^{n-s-p} a_{k}^{p+s}$.

Case 1. $x=a_{1}^{p r_{1}} a_{j}^{p r_{j}} a_{j+1}^{p r_{j+1}} \cdots a_{k-1}^{p r_{k-1}} a_{k}^{p r_{k}} a_{k}^{p+s}$ where $r_{j} \leqslant 1,2 \leqslant j \leqslant k$.

The proof is by induction on $j$, so suppose first that $j=2$. Let

$$
v=\frac{1}{(s-1) !} \times \frac{a_{1}^{p+1}}{a_{2}^{p} a_{k}^{s}} .
$$

Then $v \in\left(a_{k}^{p}\right)$. By Lemma 1.9, $\left(P^{\Delta_{k}}\right)^{s} v \equiv x$ modulo polynomials of degree less than $n+2-p$. Write $\left(P^{\Delta_{k}}\right)^{s} v-x=w+w^{\prime}$ where $w^{\prime}$ consists of the monomials whose exponents of $a_{1}$ are divisible by $p$ and $w$ consists of those whose exponents of $a_{1}$ are not divisible by $p$. Since $v \in \operatorname{Ker}\left(P^{\Delta_{k-1}}\right)^{2}, w$ does also, and so $P^{\Delta_{k-1} w} \in K$. Since 
application of $P^{\Delta_{k-1}}$ does not change polynomial degree,

$$
\begin{gathered}
d(w)=d\left(P^{\Delta_{k-1}} w\right) \equiv-\frac{1}{2}\left|P^{\Delta_{k-1}} w\right|=-\frac{1}{2}\left(|w|+2\left(p^{k-1}-1\right)\right) \\
\equiv-\frac{1}{2}|w|+1=-\frac{1}{2}|x|+1 \equiv d(x)+1 \equiv s+1 \quad(\bmod p) \\
{\left[d(w)=d\left(P^{\Delta_{k-1}} w\right) \equiv\left|P^{\Delta_{k-1}} w\right|=|w|+2^{k-1}-1\right.} \\
\equiv|x|+1 \equiv d(x)+1 \equiv 0 \quad(\bmod 2)] .
\end{gathered}
$$

Thus $w \in \operatorname{Im}\left(P^{\Delta_{k}}\right)^{s}$ and $\left(P^{\Delta_{k}}\right)^{p-s} w=0$. Therefore $\left(P^{\Delta_{k}}\right)^{p-s}\left(x+w^{\prime}\right)=$ $\left(P^{\Delta_{k}}\right)^{p-s}\left(\left(P^{\Delta_{k}}\right)^{s} v-w\right)=0$, and so $\left(P^{\Delta_{k}}\right)^{p-s}\left(u_{x}+w^{\prime}\right)=0$. But since $u_{x}$ and $w^{\prime} \in$ $K$ and $\left|u_{x}+w^{\prime}\right|=|x|$,

$$
\left(P^{\Delta_{k}}\right)^{p-s}\left(u_{x}+w^{\prime}\right)=\frac{(p-1) !}{(s-1) !} a_{k}^{p-s}\left(u_{x}+w^{\prime}\right),
$$

and since $d\left(u_{x}+w^{\prime}\right) \leqslant n-p$, the equation $\left(P^{\Delta_{k}}\right)^{p-s}\left(u_{x}+w^{\prime}\right)=0$ forces $u_{x}+w^{\prime}$ $=0$.

Let $z=(1 / s !) w / a_{k}^{s}$. Then $\left(P^{\Delta_{k}}\right)^{s} z=w$. So $\left(P^{\Delta_{k}}\right)^{s}(v-z)=x+w^{\prime}=x-u_{x}$. Thus to show $x-u_{x} \in \mathscr{F}$ it remains to show that $v-z \in \mathscr{F}$. Since $v \in\left(a_{k}^{p}\right)$, so does $w$. But $|w|=|x| \equiv s(p)$, so we must have $w \in\left(a_{k}^{p+s}\right)$ and thus $z \in\left(a_{k}^{p}\right)$. Therefore $P^{\Delta_{k-1}}(v-z) \in(a p)$. But $P^{\Delta_{k-1}}(v-z) \in K$ and

$$
\begin{gathered}
\left|\left(P^{\Delta_{k}}\right)^{s-1} P^{\Delta_{k-1}}(v-z)\right|=|x|+2\left(p^{k-1}-1\right)-2\left(p^{k}-1\right) \equiv|x| \quad(\bmod p) \\
{\left[\left|\mathrm{Sq}^{\Delta_{k-1}}(v-z)\right|=|x|+2^{k-1}-1-\left(2^{k}-1\right) \equiv|x| \quad(\bmod 2)\right] .}
\end{gathered}
$$

So $\left(P^{\Delta_{k}}\right)^{s-1} P^{\Delta_{k-1}}(v-z) \in K_{s} \cap\left(a_{k}^{p}\right)$, and thus since $\left|\left(P^{\Delta_{k}}\right)^{s-1} P^{\Delta_{k-1}}(v-z)\right|<|x|$, $\left(P^{\Delta_{k}}\right)^{s-1} P^{\Delta_{k-1}}(v-z) \in \operatorname{Ker} g$ by the induction hypothesis. But this says that $\left(P^{\Delta_{k}}\right)^{s-1} g(v-z) \in K$. Write $g(v-z)=b-b^{\prime}$ where $b$ is the sum of the monomials of $g(v-z)$ which are in $\operatorname{Ker} P^{\Delta_{k-1}}$ and $b^{\prime}$ is the sum of the other monomials. Since $\left(P^{\Delta_{k-1}}\right)^{2} g(v-z)=0$, the coefficient of $a_{1}$ in each monomial of $b^{\prime}$ is congruent to $1(\bmod p)$. However,

$$
\begin{gathered}
|g(v-z)|=|v-z|=|x|-2 s\left(p^{k}-1\right) \equiv-2 d(x)+2 s \equiv 0(p) \\
{\left[|g(v-z)|=|v-z|=|x|-\left(2^{k}-1\right) \equiv d(x)+1 \equiv 0(2)\right],}
\end{gathered}
$$

and thus the coefficient of $a_{k}$ in each monomial of $g(v-z)$ is congruent to 0 $(\bmod p)$. Hence $b$ is a $p$ th power and $d\left(b^{\prime}\right) \equiv 1(p)$, so $d\left(b^{\prime}\right) \leqslant n-s+1$. Thus $\left(P^{\Delta_{k}}\right)^{s-1} g(v-z)=(s-1) ! b^{\prime} a_{k}^{s-1}$ which is already admissible since $d\left(b^{\prime}\right) \leqslant n-s$ +1 . But $b^{\prime} a_{k}^{s-1}$ cannot be in $K$ unless $b^{\prime}=0$, and so $g(v-z)=b \in\{p$ th powers $\} \subset \mathscr{F}$. Thus $v-z \in \mathscr{F}$, as desired.

This completes the proof for $j=2$ so suppose now that the result if known for $j<i$. Set $y=x\left(a_{i-1} / a_{i}\right)^{p}$. Since $y \in K \cap\left(a_{k}^{p}\right)$ and $|y|<R, y-u_{y} \in \mathscr{F}$. Thus $P^{p\left(\left|a_{i}\right|-\left|a_{i-1}\right|\right)}\left(y-u_{y}\right) \in \mathscr{F}$. Let $w=P^{p\left(\left|a_{i}\right|-\left|a_{i-1}\right|\right)} y-x$. By Theorem 1.1 each monomial in $w$ satisfies the induction hypothesis with $j=i-1$, so $w-u_{w} \in \mathscr{F}$. Also, application of $P^{p\left(\left|a_{i}\right|-\left|a_{i-1}\right|\right)}$ cannot increase polynomial degree, so $d\left(P^{p\left(\left|a_{i}\right|-\left|a_{i-1}\right|\right)} u_{y}\right)$ $<n$. Since $P^{\Delta_{k}}$ commutes with $P^{p\left(\left|a_{i}\right|-\left|a_{i-1}\right|\right)}$,

$$
P^{\Delta_{k}} x=P^{\Delta_{k}} P^{p\left(\left|a_{i}\right|-\left|a_{i-1}\right|\right)} y-P^{\Delta_{k}} w=P^{p\left(\left|a_{i}\right|-\left|a_{i-1}\right|\right)} u_{y}-u_{w}
$$

so $u_{x}=P^{p\left(\left|a_{i}\right|-\left|a_{i-1}\right|\right)} u_{y}-u_{w}$. But then $x-u_{x}=P^{p\left(\left|a_{i}\right|-\left|a_{i-1}\right|\right)} j\left(y-u_{y}\right)-\left(w-u_{w}\right)$ $\in \mathscr{F}$, completing the induction step. 
Case 2. $x=a_{1}^{n-s-p} a_{k}^{p+s}$.

Let $y=a_{1}^{n-s-p} a_{2}^{p} a_{k}^{s}$. We first show $y-u_{y} \in \mathscr{F}$.

Since $y$ is the unique monomial of degree $n$ having total degree $|y|$, the description of $j_{n}^{*}$ in $\S 1$ shows that $Q_{\psi(y)}^{*}=y-u_{y}$. So $Q_{\psi(y)}^{*} \in \bigcap_{j=1}^{k} \operatorname{Ker} P^{\Delta_{j}}$ and thus $g Q_{\psi(y)}^{*} \in$ $\bigcap_{j=1}^{k} \operatorname{Ker} P^{\Delta_{j}}$. Since $\left|g Q_{\psi(y)}^{*}\right|=\left|Q_{\psi(y)}^{*}\right|=|y| \not \equiv 0(p), g Q_{\psi(y)}^{*}$ is not a $p$ th power so we must have $d\left(g Q_{\psi(y)}^{*}\right)=n$. Since there are no monomials of degree $n$ other than $y$ having total degree $|y|$ and $|y| \not \equiv O(p)$ so that there are no $p$ th powers in this total degree, $y-u_{y}$ generates $\bigcap_{j=1}^{k} \operatorname{Ker} P^{\Delta_{j}}$ in this total degree, so $g Q_{\psi(y)}^{*}=\lambda Q_{\psi(y)}^{*}$ for some $\lambda$. However, $Q_{\psi(y)}[1] *\left[-p^{k}\right]=e_{k-2}$ in the notation of Lemma 5.9. So Lemma 5.9 shows $\lambda=1$, which completes the proof that $y-u_{y} \in \mathscr{F}$.

Let $\alpha=P^{p} P^{p^{2}} \ldots P^{p^{k-2}}$. Then $\alpha y=x$. Since $P^{\Delta_{k}}$ commutes with $\alpha$,

$$
P^{\Delta_{k}} \alpha\left(y-u_{y}\right)=0,
$$

and since $\alpha$ cannot increase polynomial degree $\alpha u_{y}=u_{x}$, so $x-u_{x}=\alpha\left(y-u_{y}\right) \in$ $\mathscr{F}$.

Step 2. $\left(K_{s} \cap\left(a_{k}^{p}\right)\right)_{R} \cap \operatorname{Ker} P^{\Delta_{k}} \subset \mathscr{F}$.

Proof. Let $x \in\left(K_{s} \cap\left(a_{k}^{p}\right)\right)_{R} \cap \operatorname{Ker} P^{\Delta_{k}}$. Let $y$ be the sum of the monomials of $x$ having total degree $n$ and let $z=x-y$. Then $x=\left(y-u_{y}\right)+\left(z+u_{y}\right)$. Since $x$ and $y-u_{y}$ are in $\bigcap_{j=1}^{k} \operatorname{Ker} P^{\Delta_{j}}$, so is $z+u_{y}$. But $d\left(z+u_{y}\right)<n$, so $z+u_{y}$ is a $p$ th power and thus $z+u_{y} \in \mathscr{F}$. If $y$ is the sum of monomials $w_{i}$ of degree $n$, then $y-u_{y}=\sum_{i} w_{i}-u_{w_{i}}$. For each $i, w_{i}-u_{w_{i}} \in \mathscr{F}$ by Step 1 , so $y-u_{y} \in \mathscr{F}$ and thus $x \in \mathscr{F}$.

Step 3. $\left(K_{s} \cap\left(a_{k}^{p}\right)\right)_{R} \subset \mathscr{F}$.

Let $x \in\left(K_{s} \cap\left(a_{k}^{p}\right)\right)_{R}$. Then

$$
P^{\Delta_{k}} x \in K_{s+1} \cap\left(a_{k}^{p}\right) \subset \operatorname{Ker} g
$$

and so $g x \in\left(K_{s} \cap\left(a_{k}^{p}\right)\right)_{R} \cap \operatorname{Ker} P^{\Delta_{k}} \subset \mathscr{F}$ by Step 2. Thus $x \in \mathscr{F}$.

We are now ready to prove the finite loop version of our main theorem.

Proof of THeOREM 5.1. It suffices to show that $f$ induces an isomorphism on $P H^{*}\left(\Omega_{0}^{m+1} S^{m+1} ; Z / p Z\right)$ or equivalently that it induces a monomorphism since $P H^{*}\left(\Omega_{0}^{m+1} S^{m+1} ; Z / p Z\right)$ has finite type. Suppose that there is a nonzero $v \in$ $P H^{*}\left(\Omega_{0}^{m+1} S^{m+1} ; Z / p Z\right)$ such that $f^{*} v=0$. Since $v$ can have only finitely many nonzero components and $\left(R_{n}[j] / Q_{0} R_{n}[j]\right)^{*}$ is a finite dimensional vector space for each $j$, there is a nonzero $w \in \operatorname{Ker} f^{*}$ such that

$$
w \in \text { Ann } P H^{*}\left(\Omega_{0}^{m+1} S^{m+1} ; Z / p Z\right) .
$$

By Corollary 1.8 and Lemma $2.1, w \in$ Ann $W_{n}[k]^{*}$ for some $k$. To get a contradiction, thus proving the theorem, it suffices to show that for each $k$ there is an integer $N$ (depending on $k$ ) such that $\left(f[k]_{*}\right)^{N}=1$. By Lemmas 5.6 and 5.8 , this is true for $k=1$ so suppose by induction that $\left(f[k-1]_{*}\right)^{N^{\prime}}=1$. Replacing $f$ by $f^{N^{\prime}}$ we may assume that $f[k-1]_{*}=1$. Since $k$ is now fixed we will write simply $f$ for $f[k]^{*}$. Let $g$ and $\mathscr{F}$ be as in the proof of Theorem 5.3. Since $W_{n}[k]^{*}$ is finite dimensional it suffices to show $W_{n}[k]^{*}=\mathscr{F}$.

Let $z \in W_{n}[k]^{*}$. Suppose by downward induction on total degree that $y \in \mathscr{F}$ for all $y$ such that $|y|>|z|$. Replacing $f$ by $f^{p^{M}}$ for some $M$ we may assume that 
$y \in \operatorname{Ker} g$ for all $y$ such that $|y|>|z|$. Then for all Steenrod operations $\alpha, \alpha g z=$ $g(\alpha z)=0$, so $g z \in \operatorname{Ann}\left(W_{n}[k]\right)^{*}$. Since $g z \in \mathscr{F}$ implies $z \in \mathscr{F}$, it suffices to show that $\operatorname{Ann}\left(W_{n}[k]\right)^{*} \subset \mathscr{F}$. So suppose $z \in \operatorname{Ann}\left(W_{n}[k]\right)^{*}$. It suffices to consider the case where $z$ is in our basis for $\operatorname{Ann}\left(W_{n}[k]\right)^{*}$, so suppose $z=Q_{2(p-1) I}{ }^{*}\left[Q_{I}{ }^{*}\right]$ for some $Q_{2(p-1) I}\left[Q_{I}\right] \in N_{n}[k]$. Let $I=\left(i_{n}, \ldots, i_{1}\right)$ and let $x_{j}$ be as introduced after Lemma 2.1 in $\S 2$. Since $g z$ is also in $\operatorname{Ann}\left(W_{n}[k]\right)^{*}$, by Lemma $2.10 \mathrm{gz}=\lambda z$ for some $\lambda$. We must show $\lambda=0$.

If $i_{k} \geqslant p$ then $z \subset K \cap\left(a_{k}^{p}\right)$ which is contained in $\mathscr{F}$ by Theorem 5.4.

If $i_{k}<p$, consider the Nishida relation $P_{*}^{i_{k}} Q_{2(p-1) I}=x_{k-1}^{p}\left[\mathrm{Sq}_{*}^{1} Q_{I}=x_{k-2}^{2}\right]$, noting that the binomial coefficient is 1 by the properties of $N_{n}[k]$. Since $f[k-1]_{*}$ $=1, f_{*} x_{k-1} \in G$. But since $f$ is an $H$-map this implies $f_{*}\left(x_{k-1}^{p}\right) \in G$. By the properties of $N_{n}[k], x_{k-1}$ does not appear in $P_{*}^{1} Q_{2(p-1)}\left[\mathrm{Sq}_{*}^{1} Q_{J}\right]$ for any $J$ when written in the basis $B$, and so there is no element of $B$ other than $Q_{2(p-1) I}\left[Q_{I}\right]$ having the property that $P_{*}^{i_{k}} Q_{2(p-1) I}\left[\mathrm{Sq}_{*}^{1} Q_{I}\right]$ contains a nonzero multiple of $x_{k-1}^{p}$ when written in the basis $B$. Thus $P_{*}^{i_{k}} Q_{w(p-1) I}=x_{k-1}^{p}\left[\mathrm{Sq}_{*}^{1} Q_{I}=x_{k-1}^{2}\right]$ forces $Q_{2(p-1)}\left[Q_{I}\right] \in G$ and thus $\lambda=0$, completing the proof of the theorem.

Proof of Corollary 5.2. This follows immediately from Theorems 3.2 and 5.1.

\section{REFERENCES}

[A] J. F. Adams, On the non-existence of elements of Hopf invariant one, Ann. of Math. (2) 72 (1960), 20-104.

[B] J. C. Becker, Characteristic classes and K-theory, Lecture Notes in Math., vol. 428, Springer-Verlag, Berlin and New York, 1974, pp. 132-143.

[BH] S. Brewster and W. Homer, Rational automorphisms of Grassmann manifolds, Proc. Amer. Math. Soc. 88 (1983), 181-183.

[CM] F. R. Cohen and M. E. Mahowald, A remark on the self-maps of $\Omega^{2} S^{2 n+1}$, Indiana Univ. Math. J. 30 (1981), 583-588.

[CMN] F. R. Cohen, J. C. Moore and J. A. Neisendorfer, Exponents in homotopy theory (to appear).

[GH] H. Glover and W. Homer, Endomorphisms of the cohomology ring of finite Grassmann manifolds, Proc. Conf. Evanston, Ill., Lecture Notes in Math., vol. 657, I, Springer, Berlin and New York, 1978, pp. 170-193.

[H] M. Hoffman, Endomorphisms of the cohomology of complex Grassmannians (to appear).

[J] I. M. James, Spaces associated with Stiefel manifolds, Proc. London Math. Soc. (3) 9 (1959), 115-140.

[KP] D. S. Kahn and S. B. Priddy, The transfer and stable homotopy theory, Math. Proc. Cambridge Philos. Soc. 83 (1978), 103-111.

[M] J. Milnor, The Steenrod algebra and its dual, Ann. of Math. (2) 67 (1958), 150-171.

[Ma] I. Madsen, On the action of the Dyer-Lashof algebra in $H_{*}(G)$, Pacific J. Math. 60 (1975), $235-275$.

[MCL] J. P. May, F. R. Cohen and T. J. Lada, The homology of iterated loop spaces, Lecture Notes in Math., vol. 533, Springer-Verlag, Berlin and New York, 1976.

[P] F. P. Peterson, Self maps of loop spaces of spheres, Contemp. Math., vol. 12, Amer. Math. Soc., Providence, R. I., 1982, pp. 287-288.

[S1] P. S. Selick, On the indecomposability of certain sphere-related spaces, CMS Conf. Proc., vol. 2, part 1, Amer. Math. Soc., Providence, R. I., 1982, pp. 359-372.

[S2] _ A A reformulation of the Arf invariant one mod-p problem and applications to atomic spaces, Pacific J. Math. 108 (1983), 431-450.

[Se] G. B. Segal, The stable homotopy of complex projective space, Quart. J. Math. Oxford Ser. 24 (1973), 1-5. 
[Sn] V. P. Snaith, Algebraic cobordism and K-theory, Mem. Amer. Math. Soc. No. 221 (1979).

[W] C. Wilkerson, Genus and cancellation, Topology 14 (1975), 29-36.

Department of Mathematics, University of Western Ontario, London (N6A 5B9), Ontario, CANADA

Department of Mathematics, Massachusetts institute of Technology, Cambridge, MasSACHUSETTS 02139 (Current address of F. P. Peterson)

Current address (H. E. A. Campbell): Department of Mathematics, Queens University, Kingston, Ontario, Canada

Current address (P. S. Selick): Physical Sciences Division, University of Toronto, Scarborough College, Scarborough (M1C 1A4), Ontario, Canada 